\title{
Star Formation in Sculptor Group Dwarf Irregular Galaxies and the Nature of "Transition" Galaxies
}

\author{
Evan D. Skillman \\ Astronomy Department, University of Minnesota, Minneapolis, MN 55455 \\ skillman@astro.umn.edu \\ Stéphanie Côté \\ Canadian Gemini Office, HIA/NRC of Canada, 5071 West Saanich Rd., Victoria, B.C., Canada, \\ V9E 2E7 \\ Stephanie.Cote@hia.nrc.ca \\ and \\ Bryan W. Miller \\ AURA/Gemini Observatory, Casilla 603, La Serena, Chile; \\ bmiller@gemini.edu
}

\begin{abstract}
We present new $\mathrm{H} \alpha$ narrow band imaging of the HII regions in eight Sculptor Group dwarf irregular (dI) galaxies. The $\mathrm{H} \alpha$ luminosities of the detected HII regions range from some of the faintest detected in extragalactic HII regions $\left(\sim 10^{35} \mathrm{erg} \mathrm{s}^{-1}\right.$ in SC 24) to some of the most luminous ( $\sim 10^{40} \mathrm{erg} \mathrm{s}^{-1}$ in NGC 625$)$. The total $\mathrm{H} \alpha$ luminosities are converted into current star formation rates (SFR). Comparing the Sculptor Group dIs to the Local Group dIs, we find that the Sculptor Group dIs have, on average, lower values of SFR when normalized to either galaxy luminosity or gas mass (although there is considerable overlap between the two samples). The range for both the Sculptor Group and Local Group samples is large when compared to that seen for the sample of gas-rich quiescent low surface brightness (LSB) dIs from van Zee et al. (1997) and the sample of isolated dIs from van Zee $(2000,2001)$. This is probably best understood as a selection effect since the nearby group samples have a much larger fraction of extremely low luminosity galaxies and the smaller galaxies are much more liable to large relative variations in current SFR. The Sculptor Group and LSB samples are very similar with regard to mean values of both $\tau_{\text {gas }}$ and $\tau_{\text {form }}$, and the Local Group and isolated dI samples are also similar to each other in these two quantities. Presently, the Sculptor Group lacks dI galaxies with elevated normalized current SFRs as high as the Local Group dIs IC 10 and GR 8.
\end{abstract}


The properties of "transition" (dSph/dIrr) galaxies in Sculptor and the Local Group are also compared and found to be similar. The transition galaxies are typically among the lowest luminosities of the gas rich dwarf galaxies. Relative to the dwarf irregular galaxies, the transition galaxies are found preferentially nearer to spiral galaxies, and are found nearer to the center of the mass distribution in the local cloud. While most of these systems are consistent with normal dI galaxies which currently exhibit temporarily interrupted star formation, the observed density-morphology relationship (which is weaker than that observed for the dwarf spheroidal galaxies) indicates that environmental processes such as "tidal stirring" may play a role in causing their lower SFRs.

Subject headings: galaxies: dwarf — galaxies: irregular — galaxies: evolution — galaxies: individual (NGC 625) — HII regions

\section{Introduction}

Low-mass dwarf irregular galaxies provide an important testing ground for several fundamental questions about star formation, star formation rates (SFRs), galactic evolution, and cosmology. Due, in large part, to attempts to understand the possible evolutionary connections between the dwarfs with negligible or extremely low present SFRs (the dSph and dE galaxies, hereafter dE galaxies) and the dwarfs with obvious signs of present star formation (the dIrrs, blue compact dwarfs, HII galaxies, hereafter dI galaxies), many theorists are turning their attention to the problem of dwarf galaxy evolution. Environmental effects are turning out to be a key parameter (e.g., van den Bergh 1994b; Klypin et al. 1999; Moore et al. 1999; Gnedin 2000; Mayer et al. 2001a,b; Carraro et al. 2001). An especially interesting question in this regard is the effect of reionization on the suppression of dwarf galaxy formation (Efstathiou 1992; Babul \& Rees 1992; Quinn, Katz, \& Efstathiou 1996; Barkana \& Loeb 1999; Bullock et al. 2000). By comparing the properties of dwarf galaxies in different environments we may be able to isolate key environmental variables (e.g., local density, companionship, group vs. cluster membership) in order to constrain these theories.

The Sculptor group is the closest group of galaxies beyond our Local Group. Originally thought to be at a distance of $2.5 \mathrm{Mpc}$ (de Vaucouleurs 1975), the main members of the group are 5 latetype spiral galaxies (NGC 55, NGC 247, NGC 253, NGC 300, and NGC 7793) with distances ranging between 1.6 and 3.4 Mpc (Puche \& Carignan 1988 and references therein) and a dwarf spiral (NGC 45) at a distance of 4.4 Mpc. Additionally, NGC 24, another dwarf spiral, lies close to NGC 45 in radial velocity and projected location and is usually included as a group member (e.g., Giuricin et al. 2000) although some distance estimates put it as far away as 11 Mpc (Puche \& Carignan 1988). Jerjen, Freeman, \& Binggeli (1998) studied surface brightness fluctuations in 5 Sculptor group dEs and determined a spread in distances of dE members of the group from 1.7 to 4.4 Mpc (but note that Jerjen \& Rejkuba 2001 claim that some of these distances should be revised). 
A well defined relationship between radial velocity (corrected to the galactocentric standard of rest) and distance was discovered for all members of the Sculptor group (Jerjen et al. 1998), indicating that radial velocity may be a good distance indicator for Sculptor Group members. Jerjen et al. (1998) propose that the Sculptor Group and the Local Group are part of the same Supergalactic structure (a part of the Coma - Sculptor Cloud delineated by Tully \& Fisher 1987). A dynamical picture of the Sculptor Group can be found in Whiting (1999).

Miller (1994), Côté (1995), and Jerjen et al. (2000) independently searched for and found new dwarf galaxy members of the Sculptor Group. The Sculptor Group is now known to contain 16 dwarf irregular galaxies (Côté et al. 1997), some of which are amongst the lowest luminosity dwarf irregulars known. The present Sculptor Group membership situation can be found in Jerjen et al. (1998; 2000).

Miller (1996) noted that several Sculptor Group dIs were undetected in $\mathrm{H} \alpha$ emission at reasonably sensitive levels. This has several implications for the present study. First, as Miller (1996) pointed out, the Sculptor Group dIs may have relatively low average SFRs when compared to Local Group dIs. Second, although some of the Miller (1996) sample have now been detected in $\mathrm{H} \alpha$ at low levels (see §2.2), there remain three dwarf galaxies in the Sculptor Group (SDIG, DDO 6, and UGCA 438) with the properties of "transition type" galaxies (no detectable HII regions and $\mathrm{M}_{H I} / \mathrm{L}_{B}$ values in the range of $\mathrm{dI}$ galaxies). Thus, the Sculptor Group sample allows us to investigate this less common type of galaxy (see, e.g., Miller et al. 2001). Third, H $\alpha$ surveys identifying HII regions are a necessary first step in studying the ISM abundances of galaxies. Since relatively high surface brightness HII regions are usually required for an accurate abundance analysis, this means that we may not be able to accurately measure the chemical abundances in all of the known Sculptor Group dIs.

Here we present deep CCD H $\alpha$ imaging of eight Sculptor group dIs obtained at the "Danish" $1.5 \mathrm{~m}$ telescope located at the European Southern Observatory. We have detected HII regions in all eight dIs observed, in addition to the two dIs detected in $\mathrm{H} \alpha$ by Miller (1996). We present coordinates and fluxes for the HII regions and estimated SFRs for these galaxies. Additionally, we have used the CTIO 4-m to obtain optical spectra of ten HII regions located in five of these dIs and these observations are presented in a companion paper. By comparing observations of the dwarf galaxies in the next nearest group to other well defined samples, we hope to better understand basic questions such as: what are the average properties of the dwarf galaxies? and, are there observable signatures of environmental influences on galaxy evolution? 


\section{Observations}

\subsection{Target Selection}

All galaxies observed were chosen from the list of Côté et al. (1997). Table 1 lists some of the properties of the observed galaxies. Based on the observation by Jerjen et al. (1998) that the scatter in the distance - velocity relationship for the Sculptor Group members is "remarkably small", we have derived new distances for these galaxies using the recessional velocities listed in Côté et al. (1997) and the formula given in Jerjen et al. (1998):

$$
v_{\mathrm{GSR}}\left[k m s^{-1}\right]=119( \pm 7) D[M p c]-136( \pm 14) .
$$

For the nine galaxies ( 6 spirals and 3 dwarfs) used to define the relationship, the average difference between the measured distance and the estimated distance is 10\%. Eight of the nine galaxies show differences of less than 20\% (the exception being NGC 45, with a difference of $21 \%$ ). Dolphin (private communication) has calculated distances from Hubble Space Telescope observations of the red giant branch tip for five (1 spiral and 4 dwarfs) Sculptor group galaxies with known recession velocities (DDO 6, DDO 226, NGC 253, ESO 245-G005 and ESO 294-G010), and these five galaxies show good agreement with the relationship derived by Jerjen et al. (1998); all five galaxy distances are within $20 \%$ of that predicted by the relationship. The relationship should be updated with inclusion of these new data, but, based on the above, the anticipated revision will be small (of order the uncertainty in the relationship), and we will use the relationship as published. The estimated errors in distances are small enough that we can begin to examine positional relationships between galaxies. Note that the finding by Jerjen \& Rejkuba (2001) that the distances estimated for some of the dEs via the method of surface brightness fluctuations could be significantly in error does not directly imply that the formula relating distance and recessional velocity needs to be revised since most of the dEs do not have measured recession velocities and were not used in calibrating the relationship.

This equation was derived for galaxies covering the range in corrected recessional velocity from $\sim 70$ to $\sim 490 \mathrm{~km} \mathrm{~s}^{-1}$, and thus may not be strictly valid for the three galaxies observed here with corrected recessional velocities in excess of $500 \mathrm{~km} \mathrm{~s}^{-1}$. The fact that several of the dIs have recessional velocities in excess of $500 \mathrm{~km} \mathrm{~s}^{-1}$ implies that a significant number of dI galaxies lie on the far side of the Sculptor group. (Note that the spiral galaxy NGC 24, with a recession velocity of $555 \mathrm{~km} \mathrm{~s}^{-1}$ is also in this region.) In comparison with the Local Group, this is not unexpected, as several of the Local Group dIs lie in the low density periphery of the Local Group at distances in excess of $1 \mathrm{Mpc}$ from the Milky Way and M31 (Mateo 1998). The absolute B magnitudes in Table 1 were calculated from the total B magnitudes and extinctions listed in Côté (1995). From a comparison of the values in Côté (1995) and other values in the literature, the uncertainty in $\mathrm{M}(\mathrm{B})$ from the photometry is probably a little less than 0.2 magnitudes, and, when considering the error in the distance estimates, the total error is probably a little larger than 0.2 magnitudes for the typical Sculptor Group member. The error could be larger for those galaxies with velocities 
in excess of $500 \mathrm{~km} \mathrm{~s}^{-1}$. Nonetheless, these errors are small relative to the dynamic range of the luminosities of the galaxies and allow us to investigate relationships between intrinsic properties of galaxies.

\section{2. $\quad$ H $\alpha$ Imaging}

The search for HII regions in the Sculptor dIs was performed on October 24 and 25, 1995 with DFOSC at the 1.5m "Danish" telescope at the European Southern Observatory. Additional data for one galaxy (ESO 473-G24) were gathered on November 4, 1995, courtesy of Chris Lidman. The detector used was the Loral $2048 \times 2048$, giving a scale of $0.39^{\prime \prime}$ pixel $^{-1}$ and field of view of $13.6^{\prime} \times$ $13.6^{\prime}$. The CCD was read out in the high gain mode yielding a readout noise of $7.2 \mathrm{e}^{-}$r.m.s. with a gain of $1.31 \mathrm{e}^{-} / \mathrm{ADU}$. The galaxies were imaged through a narrowband $\mathrm{H} \alpha$ filter with a FWHM of $62.1 \AA$ centered at $\sim 6561 \AA$ (ESO\#693), and continuum off-band images were taken in Gunn $i$. The observations usually consisted of $2 \times 1200$ s for the $\mathrm{H} \alpha$ images and $2 \times 600$ s for the $i$-band images. The seeing varied between $\sim 1^{\prime \prime}$ and $1.3^{\prime \prime}$, but the conditions were not photometric.

The data were reduced following the usual procedures with IRAF ${ }^{1}$. After registering the different images, the two $\mathrm{H} \alpha$ and two $i$ frames were co-added. To optimize the continuum subtraction, we used a small kernel Gaussian filter to smooth the $i$ image in order to match as closely as possible the point-spread functions of the $i$ and $\mathrm{H} \alpha$ images. Half a dozen isolated bright stars were used to determine a scaling factor between the $i$ and $\mathrm{H} \alpha$ frames. The final continuum subtracted image was then produced by subtracting the appropriately scaled $i$ image from the $\mathrm{H} \alpha$ image. Figures 1a-d show the original $\mathrm{H} \alpha$ and the continuum-subtracted $\mathrm{H} \alpha$ images.

Although many HII regions are fairly well isolated, many are assembled in larger complexes, or have more complex morphologies like loops or filaments, as is commonly seen in late-type galaxies. Hence it was necessary to determine the boundaries of each HII region by eye, using POLYPHOT, selecting each distinct emission peak as a separate HII region. The borders of the HII regions were set to a constant $\mathrm{H} \alpha$ surface brightness level for each galaxy but because the nights were not photometric these levels are estimated to be varying around $5 \pm 2 \times 10^{-17} \mathrm{erg} \mathrm{cm}^{-2} \mathrm{~s}^{-1} \operatorname{arcsec}^{-2}$. The positions and fluxes of the HII regions are given in Table 2. Errors in the fluxes are estimated to be of order $20 \%$ due to photometric uncertainties, except for SC 18, SC 24, and NGC 625; these galaxies were observed at the end of the second night when the conditions had improved, and the errors are estimated to be $10 \%$. In fact, for NGC 625 , which is the only galaxy in our sample with previous $\mathrm{H} \alpha$ measurements in the literature, our fluxes agree within $4 \%$ with that of Marlowe et al. (1997). On the other hand, for AM 0106-382 the conditions had deteriorated so badly that the galaxy was not even detectable in the continuum $i$ exposures. However, our $\mathrm{H} \alpha$ images were

\footnotetext{
${ }^{1}$ IRAF is distributed by the National Optical Astronomy Observatories, which are operated by the Association of Universities for Research in Astronomy, Inc., under cooperative agreement with the National Science Foundation.
} 
compared with the $i$ data obtained by Côté (1995), and it appears that the emission peaks seen in our $\mathrm{H} \alpha$ image do not have any counterparts in the $i$ image. This indicates that they could be genuine HII regions, so they are listed as well in Table 2. None of the fluxes given in Table 2 have been corrected for [NII] contamination; these dwarf galaxies have typically very low nitrogen abundances (see companion paper), so this introduces an additional $\sim 6 \%$ flux uncertainty. For deriving accurate positions for the HII regions, HST Guide Star Reference Frame scans from the STScI Digitized Sky Survey ${ }^{2}$ were used, and were compared with positions obtained similarly by deriving astrometric plate solutions using bright stars in the Automatic Plate Measuring (APM) catalog (cf. van Zee 2000). In each case these positions agreed to within less than $2^{\prime \prime}$.

The HII regions in the two faintest galaxies (SC 18 and SC 24) were just at the limit of detectability. In both cases, coincidence with continuum sources cast doubt on whether these are true $\mathrm{H} \alpha$ sources or possible artifacts of an imperfect continuum subtraction. Further inspection and experimentation led to confidence that these are indeed real $\mathrm{H} \alpha$ sources. Nonetheless, spectroscopic observations are required for confirmation. For the present paper, we will treat these $\mathrm{H} \alpha$ sources as bona fide HII regions. Note that in the case of the Local Group dI DDO 210, van Zee et al. (1997) found a similar single $\mathrm{H} \alpha$ detection to show broad Balmer lines and no forbidden lines, and they speculate that this source may be a luminous blue variable star.

Miller (1996) observed and detected HII regions in the Sculptor group dIs UGCA 442 (= ESO 471-G06) and ESO 245-G05 (= A 143), but did not detect $\mathrm{H} \alpha$ emission from six other Sculptor group dIs. Note that we did not re-observe any of the Sculptor group dIs from Miller's sample. Miller's non-detection of HII regions in the Sculptor Dwarf Irregular Galaxy (= SDIG, ESO 349-G31) has been confirmed by Heisler et al. (1997). van Zee (2000) has observed DDO 6 (= UGCA 15) and did not detect any HII regions, but did detect a small amount of diffuse $\mathrm{H} \alpha$ emission. van Zee (2000) also observed DDO 226 (=UGCA 9) and did detect the presence of faint HII regions. Jerjen et al. (1998) detected a faint HII region in ESO 294-G010. ESO 410-G05 is an HI non-detection, and, based on HST imaging, Karachentsev et al. (2000) have determined that this is a dSph galaxy. In light of the results of the more recent deeper spectroscopy, it might be interesting to re-observe the other non-detection by Miller (1996), i.e., UGCA 438 (= ESO 407G18). Nonetheless, the claim by Miller (1996) that the average current star formation for dIs in the Sculptor group is suppressed relative to other nearby groups appears to be confirmed (see discussion in $\S 3)$.

\footnotetext{
${ }^{2}$ The Digitized Sky Surveys were produced at the Space Telescope Science Institute under U.S. Government grant NAG W-2166. The images of these surveys are based on photographic data obtained using the Oschin Schmidt Telescope on Palomar Mountain and the UK Schmidt Telescope. The plates were processed into the present compressed digital form with the permission of these institutions.
} 


\section{The HII Regions and the Star Formation Rates the Sculptor Group Dwarf Irregular Galaxies}

\subsection{The HII Region Luminosities}

As typical for dI galaxies, the majority of HII regions in these dwarfs have a random asymmetric distribution throughout the galaxies (Brosch et al. 1998; van Zee 2000). However, the most luminous HII regions are found at or near the center (in NGC 625, NGC 59 and ESO 347-G17). $\mathrm{H} \alpha$ luminosities were calculated from the $\mathrm{H} \alpha$ fluxes using the distances of Table 1 and assuming a Galactic extinction correction of the form: $\mathrm{A}(\mathrm{H} \alpha)=2.32 E(B-V)$ (Miller \& Hodge 1994), using reddening values from Schlegel et al. (1998). Figure 2 shows a histogram of the HII region luminosities for all the dwarfs. The bulk of them are mostly low luminosity, around $10^{37.5} \mathrm{erg} \mathrm{s}^{-1}$, very similar to what is found in other nearby irregulars (Youngblood \& Hunter 1999) and isolated dIs (van Zee 2000). The lowest luminosity HII region, of $10^{35.4} \mathrm{erg} \mathrm{s}^{-1}$, is found in SC 24, the nearest dwarf in our sample and also the lowest luminosity dwarf of the group. For comparison, the Orion nebula has an $\mathrm{H} \alpha$ luminosity of $10^{37} \mathrm{erg} \mathrm{s}^{-1}$ and 30 Doradus in the LMC has an $\mathrm{H} \alpha$ luminosity of $1.5 \times 10^{40} \mathrm{erg} \mathrm{s}^{-1}$ (Kennicutt 1984).

The most luminous HII regions are found in NGC 625, which is obviously undergoing an active phase of star formation, with its central region covered with bright HII regions. Its most extreme region (\#5 in Figure 1d) is actually a blend of at least 2 peaks (barely resolved with our image quality of $\left.1^{\prime \prime}\right)$, and the $\mathrm{H} \alpha$ luminosity of the whole complex $\left(4.5 \times 10^{39}\right)$ is approaching that of the supergiant HII region 30 Doradus. HII regions with these high luminosities are characteristic of nearby BCDs and are even found in a few irregulars as well (e.g., Youngblood \& Hunter 1999). In NGC 625, several arcs and filaments, typical signs of active star formation, are seen around the larger brighter complexes (regions \#4 and \#8 for example). Most interesting are the two spectacular 'chimney'-like radial filaments, extending out of the plane of the galaxy perpendicular to the major axis (regions \#11 and \#12). These structures probably trace the conduits of outflows of hot gas upward into the halo from the inner star-forming regions. The two 'chimney'-filaments extend to about $300 \mathrm{pc}$ in length, in a galaxy with a total diameter $\left(\right.$ at $\left.\mu_{B}=25 \mathrm{mag} \operatorname{arcsec}^{-2}\right)$ of $3.6 \mathrm{kpc}$. This is larger than similar chimneys traced in other galaxies with sites of intense star-formation, e.g., the irregular NGC 4449 (Bomans et al. 1997) or those seen in the Galaxy (Normandeau et al. 1996). It is expected that in low mass galaxies such as NGC 625, the conditions for massive outflows of hot gas from galactic winds should be optimized, and, indeed, Bomans \& Grant (1998) detected x-ray emission from NGC 625 with the ROSAT satellite observatory. One would expect a non-negligible fraction of the ISM to be driven out of the galaxy. High-resolution HI maps of NGC 625 show very unusual HI kinematics, with the gas in large-scale rotation around the major axis rather than the minor one (Côté et al. 2000), although no corresponding features at the location of the chimneys are seen (with the beam resolution of $15^{\prime \prime}$ ). Marlowe et al. (1997) did not detect line-splitting in $\mathrm{H} \alpha$ (a signature of expanding bubbles), but their slit position did not cover the filaments labeled regions \#11 and \#12 (and it may be that the gas is preferentially expanding along the morphological minor 
axis, which is perpendicular to the line of sight).

\subsection{Global Star Formation Rates and Timescales}

Following Kennicutt, Tamblyn, \& Congdon (1994), H $\alpha$ luminosities were converted to current SFRs with:

$$
S F R(\text { total })=\frac{L(H \alpha)}{1.26 \times 10^{41} e_{g ~ s} ~^{-1}} M_{\odot} y r^{-1}
$$

which has been derived for normal spiral galaxies with a modified Salpeter IMF. This is slightly different ( $\sim 10 \%$ lower) from the conversion proposed by Kennicutt (1983) and is the conversion adopted by Kennicutt (1998) in his grand synthesis of global SFRs in galaxies. We calculated the rates in our dwarf galaxies in the same way although no systematic adjustment was made for internal extinction (as for spiral galaxies in Kennicutt 1998) since there is a wealth of evidence that extinction is usually quite small in these low metallicity systems.

Note that using a conversion from $\mathrm{H} \alpha$ luminosity to SFR derived for normal spiral galaxies and applying it to dwarf galaxies is liable to both increased uncertainties and possible biases. The main sources of uncertainty arise from metallicity dependences of both the IMF and the production of ionizing photons by the stars. However, our main purpose here is to compare the present sample of dwarf galaxies to other samples of dwarf galaxies, and thus, it is most important to be consistent in the derivation of SFRs so that we can compare directly to other studies like that of van Zee (2001). The possible larger uncertainties and biases for the present sample will be similar to those of other studies of dwarf galaxies. Additionally, $\mathrm{H} \alpha$ measurements of star formation rates are measuring massive star formation rates and carry an implicit assumption of a constant IMF.

Table 3 shows the results, where the $\mathrm{H} \alpha$ luminosities used in Equation (2) are the sum of all the HII region luminosities in each galaxy. Summing over just the HII regions may underestimate the total $\mathrm{H} \alpha$ luminosities by neglecting diffuse $\mathrm{H} \alpha$ emission that "leaks out" of the HII regions (Hunter, Hawley, \& Gallagher 1993; Ferguson et al. 1996). Youngblood \& Hunter (1999) and van Zee (2000) note that, on average, the $\mathrm{H} \alpha$ fluxes from the sums of individual HII regions underestimate the total $\mathrm{H} \alpha$ emission by roughly a factor of two. However, van Zee (2000) points out that in low surface brightness (LSB) galaxies with low HII region coverage fractions, large aperture $\mathrm{H} \alpha$ measurements are susceptible to significant errors with even small continuum subtraction errors. Thus, in the present paper, we have chosen to simply report the sum of the $\mathrm{H} \alpha$ emission from the HII regions.

These SFRs vary over several orders of magnitude but most of them are extremely low, as the dwarfs of Sculptor are only experiencing modest star-forming activity. The SFR is known to be a strong function of absolute magnitude, for example, in the dwarf galaxies of the M81 Group (Miller \& Hodge 1994), and thus we have calculated SFRs normalized to blue luminosities in Table 3. Normalizing to L(B) significantly decreases the range, and the extreme cases on the low end (ESO 348-G09 and SC 24) and the high side (ESO 473-G24 and NGC 625) are now easily recognized. The rates for the Sculptor dIs are consistent with those of the M81 dIs for a given 
magnitude, and our values here for extremely faint dwarfs like SC 18 and SC 24 extend this trend to fainter levels.

An interesting way to characterize the present SFR is to compare it to the total gas mass and estimate a gas depletion time scale, $\left(\tau_{\text {gas }}\right)$, the number of years that a galaxy may continue to form stars at the current rate. HI gas masses and values of $\tau_{\text {gas }}$ are listed in Table 3 . As pointed out by Kennicutt et al. (1994), $\tau_{\text {gas }}$ is really a lower limit because it does not account for the gas returned to the ISM by natural stellar evolution processes. Nonetheless, the quantity $\tau_{g a s}$ is an interesting one, and the range in $\tau_{\text {gas }}$ for the Sculptor Group dIs is quite impressive. The currently star bursting NGC 625 shows a gas consumption time scale of only 3 Gyr, while several of the galaxies have consumption time scales on order or in excess of 10 times the present age of the Universe.

Our first calculation of the gas consumption time scale for the extremely quiescent galaxy SC 24 resulted in a value of $4800 \mathrm{Gyr}$, but we suspected that this was most likely due to an overestimate of its total HI gas mass. The width of the HI emission line is listed by Côté et al. (1997) as $\Delta \mathrm{V}_{20}$ $=92 \mathrm{~km} \mathrm{~s}^{-1}$, which would be an unusually large line width for such a low luminosity galaxy. The value of $\mathrm{M}(\mathrm{HI}) / \mathrm{L}(\mathrm{B})$ (23.2) was also unusually large. Inspection of the $21 \mathrm{~cm}$ spectrum published by Côté et al. (1997) showed that the detection is close to the range of Galactic emission (with a heliocentric velocity of only $79 \mathrm{~km} \mathrm{~s}^{-1}$ ) and much of the HI flux could be due to curvature in the continuum baseline that is not well fit. We inspected the HIPASS data base (Barnes et al. 2001) and found an HI detection at the position of SC 24 at a velocity of $\approx 80 \mathrm{~km} \mathrm{~s}^{-1}$, but with a $\Delta \mathrm{V}_{20} \approx$ $25 \mathrm{~km} \mathrm{~s}^{-1}$, and thus a flux integral of only $3.2 \mathrm{Jy} \mathrm{km} \mathrm{s}^{-1}$ (roughly one quarter that of the original Côté et al. (1997) value). Here we will use the HIPASS value, but interferometric HI observations of SC 24 will be necessary to truly resolve this issue. Note that with the new, lower value for the flux integral, $\mathrm{M}(\mathrm{HI}) / \mathrm{L}(\mathrm{B})(=6.29)$ is still high, and the gas consumption time scale of 1300 Gyr is still very large.

Figure 3 shows histogram comparisons of the values of $\tau_{\text {gas }}$ for the Sculptor Group dIs with three comparison groups, the Local Group dIs (from Mateo 1998), the gas-rich LSB galaxies studied by van Zee et al. (1997), and the isolated dIs of van Zee (2000, 2001). In comparison to the Local Group dIs, the distribution of Sculptor Group dIs shows a shift to larger values of $\tau_{\text {gas }}$ (although there is considerable overlap between the two samples). The range for both the Sculptor Group and Local Group samples is large when compared to that seen for the gas-rich quiescent LSB dIs and the isolated dIs. This is due, at least in part, to selection effects. The two nearby group samples have a higher proportion of extremely low luminosity galaxies, and the smaller galaxies are much more liable to large relative variations in current SFR, thus resulting in a large range in $\tau_{\text {gas }}$. Large gas content played a primary role in the selection of the LSB dIs, and a secondary role in the selection of the isolated dIs, so a bias toward larger values of $\tau_{\text {gas }}$ in these samples is expected.

Are the Sculptor Group dIs really that different from the Local Group dIs? The Local Group has two galaxies with values of $\log \left(\tau_{\text {gas }}\right) \leq 9$, and the Sculptor Group has three galaxies with $\log$ 
$\left(\tau_{\text {gas }}\right) \geq 11.5$, so, at least statistically these two distributions could be found to be different. Using the Kolmogorov-Smirnov (hereafter K-S) test (specifically kstwo of Press et al. 1992) to determine the significance of the differences between the distributions in Figure 3, we find that the probability that the Sculptor Group and Local Group galaxies are drawn from the same distributions is 30\%. This emphasizes the large degree of overlap in the two samples. Interestingly, the K-S test yields a probability of $89 \%$ that the Sculptor Group and LSB samples are drawn from the same distribution. However, this reflects the fact that the K-S test has a higher sensitivity to the median values of distributions than to the spreads in distributions (Press et al. 1992); the standard deviations of the two samples differ by a factor of two. The F-test statistic (ftest from Press et al. 1992) indicates that the Sculptor Group and the LSB dIs have significantly different variances (significance of 99\%). All other K-S test comparisons of the samples shown in Figure 3 yield probabilities of less than $10 \%$. Note that since the current star formation rates represent a snapshot in time, we have no guarantee that these distributions might not have looked very different just a few $10^{8}$ years ago.

Another important caveat should be added at this point; missing from Figure 3 are gas-rich galaxies with current star formation rates of zero (no $\mathrm{H} \alpha$ emission). The Local Group has five of these dIrr/dSph or "transition" galaxies (Mateo 1998) and the Sculptor Group has three. Although not plotted in Figure 3, the consideration of these galaxies would increase the degree of overlap in the two samples. The transition galaxies are discussed in more detail in $\S 4$.

There are different physical reasons for a galaxy to have a very large value of $\tau_{\text {gas }}$. For example, van Zee et al. (1997) and Kennicutt \& Skillman (2001) found values in excess of 200 Gyr for the LSB dI DDO 154. This appears to be mainly due to the very large HI halo surrounding this galaxy which contains $90 \%$ of the galaxy's HI. In this case, most of this gas is probably unavailable for star formation. In the case of episodic star formation, it is possible to observe a galaxy with a current SFR that is well below its average SFR. This results in an artificially large value of $\tau_{\text {gas }}$. With detailed recent star formation histories like those available from imaging studies of the young stellar population (e.g., Dohm-Palmer et al. 1997, 1998) it is possible to get a better measure of the average recent SFR.

Often the $\mathrm{H} \alpha$ and total luminosities of galaxies are used in order to investigate how the current SFR compares to some measure of the past average SFR. This ratio has been referred to as both the "star formation timescale" (Roberts 1963, Hodge 1993) which is the ratio of the mass of the stars present to the current rate of star formation and the "birthrate parameter" (Scalo 1986, Kennicutt et al. 1994) which is the ratio of the past average SFR to the current SFR. Deriving the past average SFR requires estimating the total mass of the stars formed over the lifetime of the galaxy. This can be done by converting the color of the stellar population into a representative mass-to-light ratio (e.g., Miller 1996). Alternatively, Kennicutt et al. (1994) convert the H $\alpha$ equivalent width directly into a birthrate parameter. Both methods depend on assumptions of stellar and galaxy evolution, and making assumptions about galaxy evolution in the study of galaxy evolution may produce misleading results (see discussion in van Zee 2001). Often these models are constructed with large, solar metallicity spiral galaxies in mind, so the direct applicability to low metallicity 
dwarfs carries an additional uncertainty.

Here we will follow Hodge (1993) and make the simple assumption of a mass-to-blue light ratio of 1 so that the ratio of the current SFR to the past average SFR $\left(\tau_{\text {form }}\right)$ is directly comparable to the values listed in Hodge (1993). Assuming a single value of M/L for all of the galaxies is partially supported by the small range in colors observed for these galaxies. From the photometry of Côté (1995), we find that all but two of the galaxies listed in Table 3 have values of $\mathrm{B}-\mathrm{R}$ between 0.7 and 1.0 (with ESO 471-G06 slightly bluer at 0.6 and ESO 245-G05 even bluer at 0.3). Thus, $\tau_{\text {form }}$ is simply $\mathrm{L}(\mathrm{B}) / \mathrm{SFR}$ with both given in solar units. This is listed in Table 3, column 4, which is simply the inverse of the values in column 3. Hodge (1993) studied the sample of galaxies in Kennicutt (1983) and found average values of $\tau_{\text {form }}$ of about 60 Gyr for early type spirals, 15 Gyr for later type spirals and 8 Gyr for irregular galaxies. The large values of $\tau_{\text {form }}$ found here indicate that the typical $\mathrm{dI}$ in the Sculptor Group is currently forming stars at a much lower rate than it has in the past, in support of the earlier finding by Miller (1996).

Figure 4 shows histogram comparisons of the values of $\tau_{\text {form }}$ for the Sculptor Group dIs with the same three comparison groups used in Figure 3. As in Figure 3 where $\tau_{\text {gas }}$ is compared, the range for $\tau_{\text {form }}$ in the Sculptor Group dI sample is shifted to larger values relative to the Local Group dI sample (again with considerable overlap between the two samples). The Local Group sample stands out with three galaxies with relatively low values of $\tau_{\text {form }}$ (IC 10, GR 8, and NGC 6822). Once again, this is due, in part, to selection effects. The gas-rich quiescent LSB dI sample and the isolated dI sample excluded galaxies with exceptionally high values of current SFR (HII galaxies or blue compact dwarf galaxies). It is also interesting to note the suggestion that IC 10 should be considered to be a blue compact galaxy (Richer et al. 2001 and references therein).

Are the Sculptor Group and Local Group dI distributions different in Figure 4? Here the K-S test gives a probability of only 10\% that the Local Group and Sculptor Group samples are drawn from the same distribution. This is due, in part, to the presence of IC 10 and GR 8 in the Local Group sample. If these two galaxies are deleted from the Local Group sample, then the K-S test probability rises to $22 \%$, on the margin of a significant difference. However, IC 10 and GR 8 do exist, so it makes no sense to delete them from the sample. The K-S test yields a $71 \%$ probability for the Sculptor Group sample and the LSB sample to be drawn from the same distribution. The Sculptor Group and LSB samples are very similar with regard to mean values of both $\tau_{g a s}$ and $\tau_{\text {form }}$, while the the Local Group and isolated dI samples are also similar to each other in these two quantities.

Interestingly, the K-S test of the Figure 4 data reveals that the Local Group dI sample has a $51 \%$ probability of being drawn from the same distribution as the isolated dI sample, and a $15 \%$ probability when compared to the LSB dI sample. These are significantly higher than the respective $\tau_{\text {gas }}$ comparisons ( $8 \%$ and $5 \%$ respectively). In fact, the Local Group and isolated dI sample distributions in Figure 4 do not look that similar, and the high significance once again reflects the fact that the K-S test has a higher sensitivity to the median values of distributions than 
to the spreads in distributions.

In Figure 5 we compare the two timescale values $\left(\tau_{\text {gas }}\right.$ and $\left.\tau_{\text {form }}\right)$ to each other for the four samples. Figure 5 shows a general correlation of $\tau_{\text {gas }}$ with $\tau_{\text {form }}$ (as should be expected since both axes share a common denominator). The main reason for the relatively good correlation in Figure 5 is that the gas-rich dwarf galaxies generally show a limited range in $\mathrm{M}(\mathrm{HI}) / \mathrm{L}(\mathrm{B})$ (Skillman 1996). The dotted line in Figure 5 represents an equality between $\tau_{\text {gas }}$ and $\tau_{\text {form }}$, or, equivalently, $\mathrm{M}(\mathrm{HI}) / \mathrm{L}(\mathrm{B})=1.0$. The conclusions of Figure 3 and 4 are supported. While the Sculptor Group dIs and the Local Group dIs tend to fall into the region of the graph that is well populated by the larger LSB and isolated dI samples, it is interesting that the Sculptor group dIs lie at preferentially higher values of $\log \left(\tau_{\text {gas }}\right)$ with the majority above 10.5, while the Local Group dIs lie at preferentially lower values of $\log \left(\tau_{\text {gas }}\right)$ with the majority below 10.5. It is interesting that, when normalized by SFR, the LSB galaxies do not deviate far from the trend. Naively, one might expect them to deviate to the upper right (higher gas content and lower luminosity), but, in fact, they do not appear to distinguish themselves in this diagnostic diagram. This is simply due to the fact that the dynamic range in the SFR is much larger than the dynamic range in $\mathrm{M}(\mathrm{HI}) / \mathrm{L}(\mathrm{B})$.

Finally, in Figure 6, we have plotted the SFR versus gas mass with both normalized to the galaxy luminosity $\left(\mathrm{SFR} / \mathrm{L}_{B} \text { versus } \mathrm{M}_{H I} / \mathrm{L}_{B}\right)^{3}$. In this diagnostic diagram excursions are much more prominent. The high relative SFR galaxies from the Local Group are not unusually gas rich. The Sculptor Group galaxies tend toward larger gas fractions and lower values of SFR (note the large number of them in the lower right quadrant). The isolated dIs show a large range in values, reaching toward the extremes seen in the two group samples. It would appear that the isolated dIs sample of van Zee $(2000,2001)$ covers the range of properties of dIs with the possible exception of the lowest luminosity systems.

\section{Comparing "Transition" Galaxies and dI Galaxies}

Of course, the very large values of $\tau_{\text {gas }}$ seen in several of the Sculptor Dwarf dIs do not represent an upper bound; there are several Sculptor Group dIs with HI detected but no H $\alpha$ emission detected, and these would have $\tau_{\text {gas }}$ values of infinity. Such galaxies are not limited to the Sculptor Group; in the Local Group we have LGS-3, Antlia, and DDO 210 as examples of dIs with detectable HI but no detectable $\mathrm{H} \alpha$ emission (Mateo 1998). These galaxies are often referred to as "transition" galaxies or dIrr/dSph galaxies ${ }^{4}$. Mateo (1998) also includes Pegasus and Phoenix in

\footnotetext{
${ }^{3}$ The value of $\mathrm{M}(\mathrm{HI}) / \mathrm{L}(\mathrm{B})$ for SagDIG in Table 4 of Mateo (1998) of 8.6 is in error, and we use the corrected value of 0.86 here.

${ }^{4}$ Note that this definition of "transition" dwarf galaxy is different from of Sandage \& Hoffman (1991) which was used to characterize galaxies whose optical appearances showed both early and late-type dwarf characteristics. Knezek, Sembach, \& Gallagher (1999) have studied the transition galaxies of Sandage \& Hoffman (1991) and have found them to be a rather heterogeneous sample.
} 
this category. Pegasus has two detected HII regions (Skillman, Bomans, \& Kobulnicky 1997) and the formal calculation of $\tau_{\text {gas }}$ for Pegasus yields a value of $3220 \mathrm{Gyr}^{5}$. Until recently, an optical radial velocity was unavailable for Phoenix, and it was not clear whether the HI detected in the direction of Phoenix was directly associated with it (Carignan, Demers, \& Côté 1991; Oosterloo et al. 1996, Young \& Lo 1997, St-Germain et al. 1999). However, Gallart et al. (2001) have now provided an optical radial velocity of $-52 \pm 6 \mathrm{~km} \mathrm{~s}^{-1}$, which is relatively close to the HI cloud separated from Phoenix by $6^{\prime}$ with a velocity of $-23 \mathrm{~km} \mathrm{~s}^{-1}$, and they conclude that the properties of this HI cloud are consistent with having been recently lost by Phoenix. The recent measurement of the optical radial velocity of Phoenix of $-13 \pm 9 \mathrm{~km} \mathrm{~s}^{-1}$ by Irwin \& Tolstoy (2002) strengthens the connection between the HI cloud and the galaxy. Note that the stellar population study by Holtzman, Smith, \& Grillmair (2000) indicates that Phoenix has experienced star formation up until roughly 100 million years ago which implies that Phoenix must have had some gas until very recently.

For the galaxies with the very low values of SFR (or high values of $\tau_{\text {gas }}$ and $\tau_{\text {form }}$ ), the precise value of the current SFR is probably not very meaningful. The conversion from $\mathrm{H} \alpha$ flux to SFR calculated by Kennicutt et al. (1994) is based on a fully populated IMF, and with so few HII regions, it is clear that the whole range of massive stars is not represented. Since the presence or absence of a few HII regions can move an extremely low luminosity galaxy between the dI and transition categories, they may have much in common. One also has to consider the uncertainty introduced by the differences between the $\mathrm{H} \alpha$ fluxes calculated from the sum of the HII regions and those calculated from the entire image (including the diffuse component) which can be significant for these very quiescent galaxies.

There are at least two different evolutionary paths that a dI galaxy can take to become a transition galaxy. One possibility is for the galaxy to lose enough of its cold gas to halt present star formation. A second possibility is to have sufficient gas for star formation, but to be simply "in between" episodes of star formation. Phoenix may be a local example of the first case (as discussed by Gallart et al. 2001), while Antlia, DDO 210, LGS-3, and Pegasus may be examples of the second case. Typically, dI galaxies have $\mathrm{M}_{H I} / \mathrm{L}$ ratios of about one in solar units (Skillman 1996). Thus, galaxies with similar $\mathrm{M}_{H I} / \mathrm{L}$ but no current star formation could be what would normally be called dIs, but are simply between episodes of galaxy formation. Simply based on the number of stars and the average lifetimes of HII regions, the smaller the dI galaxy, the higher the likelihood that it could be found in such a phase. Note that all five of the Local Group transition galaxies have absolute V magnitudes near the low end of the dI luminosity function (DDO $210=-10.0$, Phoenix $=-10.1$, LGS-3 $=-10.5$, Antlia $=-10.8$, and Pegasus $=-12.9$; Mateo 1998). Of the five Local Group transition galaxies, all have significant $\mathrm{HI}$ contents with values of $\mathrm{M}_{H I} / \mathrm{L}_{V}$ close to one (DDO 210

\footnotetext{
${ }^{5}$ This calculation uses the $\mathrm{H} \alpha$ flux of the HII regions. If one uses the total $\mathrm{H} \alpha$ flux reported by Hunter et al. (1993), then the value of $\tau_{\text {gas }}$ decreases to 252 Gyr. Values for Pegasus are not plotted in Figures 3, 4, 5, \& 6 because the SFR rate listed in Mateo (1998) is 0.
} 
$=2.35$, Phoenix $=0.21$, LGS-3 $=0.32$, Antlia $=0.56$, and Pegasus $=0.45$; Mateo 1998); the value for Phoenix represents the candidate HI cloud at $-23 \mathrm{~km} \mathrm{~s}^{-1}$. Thus, four of the transition galaxies in the Local Group are consistent with the evolutionary scenario of temporarily interrupted star formation.

In a detailed study of LGS-3, Miller et al. (2001) have shown how the SFR in the outer parts of LGS-3 has decreased faster than the SFR in the inner parts. If the "active" star forming area of a galaxy decreases with time, then the likelihood that it will experience a "transition" phase with no current massive star formation will increase with time. Nonetheless, it may retain sufficient gas for future episodes of star formation.

The Sculptor Group, with three galaxies with clear transition type properties, provides us with additional case studies of transition type galaxies. SDIG, DDO $6^{6}$, and UGCA 438 are all HII region non-detections with $\mathrm{HI}$ detections and $\mathrm{M}_{H I} / \mathrm{L}_{B}$ values close to one (0.88, 1.4, and 2.4 respectively). ESO 294-G10 is an HI non-detection from both Côté et al. (1997) and the HIPASS database, but has an HII region observed (Jerjen et al. 1998), so it might be interesting to re-observe it in HI. Additionally, four of the galaxies in Table 3 have values of $\tau_{\text {gas }}$ larger than 100 Gyr with values of $\mathrm{M}_{H I} / \mathrm{L}_{B}$ typical for dIs (ESO 471-G06, ESO 348-G09, SC 18, and SC 24).

Although there has not been a great deal of research on transition type galaxies, the fact that they (and galaxies with similar properties) turn up frequently in the Local Group (5 examples) and Sculptor Group (3 examples) indicates that this type of galaxy may be very common among the lowest luminosity, gas-rich, star forming galaxies. It is then an interesting question whether the spatial distribution of transition type galaxies in our Local Group and the Sculptor Group is different from the spatial distribution of normal dIs. In Figure 7 we plot the spirals, dIs, and transition galaxies from the Local and Sculptor groups in Supergalactic coordinates (cf. Jerjen et al. 1998, Figure 9). The distances for the spirals are taken from Jerjen et al. (1998), but we have added NGC 24 at a distance corresponding to Equation (1). The dEs, which tend to cluster near the spiral galaxies, are left out for clarity. Inspection of Figure 7 shows no obvious pattern to distinguish the dIs from the transition galaxies. If the low SFRs in the transition galaxies were due to the influence of the large spiral galaxies, we might expect to see evidence of a morphology density relationship like that observed for the dE galaxies (e.g., Binggeli, Tarenghi, \& Sandage 1990 and references therein), but none is immediately evident in Figure 7.

However, Figure 8 shows the same galaxies in another projection, that of the Supergalactic $\mathrm{X}, \mathrm{Z}$ plane. By coincidence, the Sculptor Group is nearly centered on the Supergalactic Y-axis (de Vaucouleurs 1958, 1975). Thus, if the Local Group - Sculptor Group concentration is modeled as a cylinder like filament aligned along the Supergalactic Y-axis, this projection shows the typical displacement from the center of that concentration. Figure 8 gives the appearance that the transi-

\footnotetext{
${ }^{6}$ Note the ambiguity for DDO 6 in that $\mathrm{H} \alpha$ emission is detected, but HII regions are not identified. We have included it as a transition galaxy because no HII regions are detected with the somewhat arbitrary justification that $\mathrm{H} \alpha$ emission can arise from sources other than photoionization from young massive stars.
} 
tion galaxies show a concentration to the center of this filament similar to that seen by the spirals, while the dIs appear to have larger radial distances.

Figure 9 is a histogram of the radial distances from this axis sorted by galaxy type. Average radial distances and standard deviations have been calculated for each of the types. Here we are including the $\mathrm{dE}$ galaxies for the first time. The distances for the Local Group $\mathrm{dE}$ galaxies are taken from Mateo (1998) and van den Bergh (2000). The distances of the six known Sculptor Group dEs come from Jerjen et al. (1998: NGC 59, SC 22, ESO 294-G010, ESO 540-G030), Karachentsev et al. (2000: ESO 410-G005), and Jerjen \& Rejkuba (2001: ESO 540-G032). The histogram ${ }^{7}$ confirms the impression that the radial distributions of the transition galaxies are very similar to those of the spiral galaxies and $\mathrm{dE}$ galaxies and distinctly different from those of the dIs. If the galaxies are separated into the Local and Sculptor groups, the offset is still present. The main difference is the significant population of dIs more than $1 \mathrm{Mpc}$ from the cloud axis. The bimodality of the $\mathrm{dE}$ distribution simply reflects the fact that most of these galaxies are either satellites of M 31 (with an offset from the Supergalactic Y-axis of $0.7 \mathrm{Mpc}$ ) or the Milky Way (with no offset).

Figure 10 shows the results of the calculation of the distances of the dI, transition, and $\mathrm{dE}$ galaxies to the nearest spiral galaxy inspired by the results of Figures 8 and 9 . In this figure, the Sculptor Group dwarfs are noted by dots in the histograms ${ }^{8}$. The main difference between the $\mathrm{dI}$ and transition galaxies in Figure 10 is the lack of transition galaxies at large distances, with the result that the average distance for the dIs is almost a factor of two larger than that of the transition galaxies. This is a marginally stronger result from that shown in Figure $9(0.82 / 0.58=$ a factor of 1.4 for average radial distance from the Cloud axis versus $0.92 / 0.50=$ a factor of 1.9 for average distance to the nearest spiral). The K-S test indicates that all three distributions in Figure 10 are significantly different. Comparing the transition galaxies to the other two samples yields only a $15 \%$ probability that the transition galaxies come from the same sample as the dIs and only an $8 \%$ probability of coming from the same sample as the dEs.

The distances calculated for Figure 10 carry a larger degree of uncertainty than those in Figure 9 since the distances to most of the Sculptor dwarfs are based on the distance-velocity relationship in Equation (1). Nonetheless, it is natural to ask the question whether the transition galaxies are found preferentially near to the spiral galaxies or to the axis of the Local Group - Sculptor Cloud that is defined by the spiral galaxies. On its own, the near identity of the means and standard deviations of the transition galaxy distances to the group axis $(0.58,0.24)$ and the nearest spiral

\footnotetext{
${ }^{7}$ Note that in constructing this histogram, NGC 55 appears twice because it is generally referred to as a late type spiral member of the Sculptor Group (SB(s)m; de Vaucouleurs, de Vaucouleurs, \& Corwin 1976), but listed as a dI (Irr IV; van den Bergh 1994a) member of the Local Group by Mateo (1998).

${ }^{8}$ Note that as a result of the inclusion of NGC 55 in our list of Sculptor spirals, two of the Local Group dIs listed by Mateo have NGC 55 as their nearest neighbor spiral, and therefore are marked with dots. These two galaxies are IC 5152 and UKS2323-326. Because of its listing as both a Sculptor spiral and a Local Group dI, NGC 55 shows up as a nearest neighbor to itself, but we have excluded that from the histogram.
} 
$(0.50,0.34)$ prevent us from distinguishing which effect may be more relevant for the evolutionary status of these galaxies. Of course, the results for the nearest spiral galaxy distances may change as better distances become available for the bulk of the sample.

In comparing the transition galaxies to the $\mathrm{dE}$ galaxies we find a significant difference between the distributions in Figures 9 and 10. The ratio of the average distance to the nearest spiral (2.3 times larger for the transition galaxies relative to the $\mathrm{dE}$ galaxies) is much larger than the ratio of the average distance to the group axis (1.5 times larger for the transition galaxies). However, this is mainly reflecting the offset of M 31 (and therefore its companion dE galaxies) from the group axis. Although the samples are admittedly small, the impression is that the three dwarf morphological types are separable by mean distance to nearest neighbor spiral galaxy. This would support the hypothesis that a key factor affecting dwarf morphological type is environmental.

There is, of course, the possibility that Figure 10 is susceptible to the biases of selection effects. It is a safe bet that there remain low luminosity, LSB galaxies undetected in the Sculptor Group. Recently, most of the new low luminosity dEs in the Local Group have been found by searching in the vicinity of M 31 (e.g., Armandroff, Jacoby, \& Davies 1999), and this might skew the distribution of Local Group dEs towards higher proximity. Additionally, the Sculptor dEs do not show the same degree of proximity to the spiral galaxies as the Local Group dEs. In the histogram in Figure 10, the Sculptor Group dEs (marked by dots) account for 4 of 23 of the galaxies in the main clump and 2 of 4 of the outliers. On the other hand, the distances to the Sculptor Group dEs are much more uncertain, and this may introduce some scatter. For example, for ESO 540-G030 and ESO 540-G032, the calculated nearest spiral neighbor (NGC 247) distances are $560 \mathrm{kpc}$ and 670 $\mathrm{kpc}$ respectively, but the minimum projected distances are $120 \mathrm{kpc}$ and $50 \mathrm{kpc}$. Ironically, the surface brightness fluctuation distance for ESO 540-G032 placed it very close to NGC 247, but the newer tip of the red giant branch measurement moved it over $1 \mathrm{Mpc}$ further away, so that its nearest neighbor changed to NGC 253. It could be that the distances are still quite uncertain, and that all of the Sculptor dEs could lie in the main group in the histogram determined by the Local Group dEs. Hubble Space Telescope distances from tip of the red giant branch measurements for all of these galaxies are required to place this comparison on firmer ground.

\section{The Nature of "Transition" Galaxies}

The present data indicate that, in the local cloud, transition galaxies are differentiated from dIs by their, on average, closer proximity to spiral galaxies. Since transition galaxies are preferentially found to be lower luminosity systems than the average dI galaxy, a luminosity - distance relationship for the dIs might cause such difference. Indeed, Armandroff et al. (1999) show that the extreme low luminosity dSph galaxies in the Local Group are found preferentially close to our Milky Way galaxy. However, this does not appear to be the case for the Local Group dIs, since the Local Group has several relatively low luminosity dIs at large distances $(\geq 1 \mathrm{Mpc})$ from the nearest spiral galaxies. This points to an underlying cause more closely related to environment (something is happening 
to these galaxies) as opposed to initial conditions (it is not true that smaller dI galaxies are formed closer to spirals).

What can be made of the morphology-density relationship for the transition galaxies? The transition galaxies do not show the same degree of morphology-density relationship as the dwarf spheroidals (i.e., the typical transition galaxy is not a close $(\leq 200 \mathrm{kpc})$ companion to a large spiral galaxy). Therefore, explanations of the morphology-density relationship applicable to the dwarf spheroidals are not necessarily appropriate for the transition galaxies. Because there is a large degree of overlap in the spatial distributions of the transition galaxies and the dIs, it is likely that the typical transition galaxy represents the extreme in the distribution of low SFRs of quiescent $\mathrm{dI}$ galaxies. The observation that the transition galaxies tend toward the center of the filamentary group structure, and lie closer, on average, to the spiral galaxies, may be telling us that environmental effects have driven otherwise normal dI galaxies to the observed low values of SFR.

Perhaps these transition galaxies have a higher probability for "tidal stirring" or "harassment" than the typical dI, resulting in their partial evolution to the dwarf spheroidal state (Mayer et al. 2001a,b). Although such interactions result in a temporarily elevated SFR, the main long-term effect of these interactions is to strip the galaxy of gas, resulting in lower SFRs. Given the small samples involved, and the limited range in environment that has been sampled here, definitive statements cannot be made. It is not clear whether galaxy luminosity (small galaxies are most easily found in a quiescent state), position relative to the "cloud" structure (galaxies near the centers of large scale structures are most likely to have the properties of transition galaxies), or position relative to the nearest spiral galaxy (galaxies nearest to spiral galaxies are most likely to be tidally stirred) is the dominant variable in determining that a gas-rich dwarf has the properties of a transition galaxy. Similar studies of other environments should provide additional constraints on this problem. Additionally, clearer observational predictions from the models would be helpful. Can tidally stirred galaxies be differentiated from isolated galaxies by bursty star formation histories or strong stellar population radial gradients? Can a burst of star formation triggered by an external influence be distinguished from a burst with an internal trigger?

Finally, while our conclusion that the transition galaxies have more in common with the dI galaxies than with the $\mathrm{dE}$ galaxies may be debatable, it is clear that transition galaxies are not $\mathrm{dE}$ galaxies. The similarities between transition galaxies and $\mathrm{dI}$ galaxies in gas content and spatial distribution may make it natural to group the transition galaxies with the dIs in making comparative family studies. Note that this is the opposite of the assumption of Blitz \& Robishaw (2000) in their study of "gas-rich dwarf spheroidals." By including the transition galaxies in with the dwarf spheroidals, they concluded that a large fraction of the dwarf spheroidals in the Local Group are associated with HI gas. We would argue that the transition galaxies should be excluded from this sample, leading to the opposite conclusion, that only a very small fraction (if any) of dwarf spheroidals in the Local Group are associated with HI gas. 


\section{Conclusions}

We have presented $\mathrm{H} \alpha$ imaging of dI galaxies in the nearby Sculptor Group. Comparing the Sculptor Group dIs to the Local Group dIs, in support of Miller (1996), we find that the Sculptor Group dIs have, on average, lower values of SFR when normalized to either galaxy luminosity or gas mass (although there is considerable overlap between the two samples). The range for both the Sculptor Group and Local Group samples is large when compared to that seen for the sample of gas-rich quiescent LSB dIs from van Zee et al. (1997) and the sample of isolated dIs from van Zee $(2000,2001)$. This is probably best understood as a selection effect since the nearby group samples have a much larger fraction of extremely low luminosity galaxies and the smaller galaxies are much more liable to large relative variations in current SFR. The Sculptor Group and LSB samples are very similar with regard to mean values of both $\tau_{\text {gas }}$ and $\tau_{\text {form }}$, and the the Local Group and isolated dI samples are also similar to each other in these two quantities.

The properties of "transition" galaxies in Sculptor and the Local Group are also compared and found to be similar. The transition galaxies are preferentially among the lowest luminosities of the gas rich dwarf galaxies. Relative to the dwarf irregular galaxies, the transition galaxies are found preferentially nearer to spiral galaxies, and are found nearer to the center of the mass distribution in the local cloud. However, the transition galaxies are not found as close to the nearest neighbor spiral galaxies as the $\mathrm{dE}$ galaxies. Most of these systems are consistent with normal dI galaxies which currently exhibit temporarily interrupted star formation. The observed density-morphology relationship indicates that environmental processes such as "tidal stirring" may play a role in causing their lower SFRs.

Special thanks are extended to Chris Lidman for acquiring the ESO 473-G24 H $\alpha$ observations, and to Liese van Zee for the use of her astrometry package to derive the HII region positions. We wish to thank G. Bothun, R. Kennicutt, E. Tolstoy, and L. van Zee for many helpful conversations. John Cannon, Henry Lee, and Liese van Zee proofread and provided valuable comments on this manuscript. We also thank the referee for a prompt and careful reading of the manuscript and several valuable comments. This research has made use of the NASA/IPAC Extragalactic Database (NED) which is operated by the Jet Propulsion Laboratory, California Institute of Technology, under contract with the National Aeronautics and Space Administration. This research has made use of NASA's Astrophysics Data System Abstract Service. EDS acknowledges partial support from a NASA LTSARP grant No. NAG5-9221 and the University of Minnesota. BWM is supported by the Gemini Observatory, which is operated by the Association of Universities for Research in Astronomy, Inc., on behalf of the international Gemini partnership of Argentina, Australia, Brazil,

Canada, Chile, the United Kingdom, and the United States of America. We would like to dedicate this paper to the memory of Robert A. Schommer, whose many contributions to the astronomical community, and the Chilean astronomical community in particular, will always be appreciated. 


\section{REFERENCES}

Armandroff, T. E., Jacoby, G. H., \& Davies, J. E. 1999, AJ, 118, 1220

Babul, A. \& Rees, M. J. 1992, MNRAS, 255, 346

Barkana, R., \& Loeb, A. 1999, ApJ, 523, 54

Barnes, D. G. et al. 2001, MNRAS, 322, 486

Binggeli, B., Tarenghi, M., \& Sandage, A. 1990, A\&A, 228, 42

Blitz, L. \& Robishaw, T. 2000, ApJ, 541, 675

Bomans, D.J., Chu, Y.H., \& Hopp, U. 1997, AJ, 113, 1678

Bomans, D.J., \& Grant, M.-B. 1998, Astron. Nach., 319, 26

Brosch, N., Heller, A., \& Almoznino, E. 1998, MNRAS, 300, 1091

Bullock, J.S., Kravtsov, A.V., \& Weinberg, D.H. 2000, ApJ, 539, 517

Carraro, G., Chiosi, C., Girardi, L., \& Lia, C. 2001, MNRAS, 327, 69

Carignan, C., Demers, S. \& Côté, S. 1991, ApJ, 381, L13

Côté, S. 1995, Ph.D. Thesis, Australian National University

Côté, S., Carignan, C., \& Freeman, K.C. 2000, AJ, 120, 3027

Côté, S., Freeman, K. C., Carignan, C., \& Quinn, P. 1997, AJ, 114, 1313

de Vaucouleurs, G. 1958, AJ, 63, 253

de Vaucouleurs, G. 1975, in Stars and Stellar Systems 9, Galaxies and the Universe, ed. A. Sandage, M. Sandage, \& J. Kristian (Chicago: Univ. Chicago Press), 557

de Vaucouleurs, G., de Vaucouleurs, A., Corwin, H. G. Jr., Buta, R. J., Paturel, G., \& Foqué, P. 1991, Third Reference Catalog of Bright Galaxies, (New York: Springer) (RC3)

Dohm-Palmer, R. C. et al. 1997, AJ, 114, 2527

Dohm-Palmer, R. C. et al. 1998, AJ, 116, 1227

Efstathiou, G. 1992, MNRAS, 256, 43P

Ferguson, A. M. N., Wyse, R. F. G., Gallagher, J. S., \& Hunter, D. A. 1996, AJ, 111, 2265

Gallagher, J. S., Tolstoy, E., Dohm-Palmer, R. C., Skillman, E. D., Cole, A., Hoessel, J., Saha, A., \& Mateo, M. 1998, AJ, 115, 1869

Gallart, C., Martinez-Delgado, D., Gomez-Flechoso, M.A., Mateo, M. 2001, AJ, 121, 2572

Gnedin, N. 2000, ApJ, 535, L75

Giuricin, G., Marinoni, C., Ceriani, L., \& Pisani, A. ApJ, 543, 178

Heisler, C.A., Hill, T.L., McCall, M.L., Hunstead, R.W. 1997, MNRAS, 285, 374 
Hodge, P. 1993, in Star Formation, Galaxies, and the Interstellar Medium, eds. J. Franco, F. Ferrini, \& G. Tenorio-Tagle, Cambridge University Press, 294

Holtzman, J. A., Smith, G. H., \& Grillmair, C. 2000, AJ, 120, 3060

Hunter, D. A., Hawley, W. N., \& Gallagher, J. S. 1993, AJ, 106, 1797

Irwin, M. \& Tolstoy, E. 2002, MNRAS, 336, 643

Jerjen, H., Freeman, K. C., \& Binggeli, B. 1998, AJ, 116, 2873

Jerjen, H., Binggeli, B., \& Freeman, K. C. 2000, AJ, 119, 593

Jerjen, H., \& Rejkuba, M. 2000, A\&A, 371, 487

Karachentsev, I. D. et al. 2000, ApJ, 542, 128

Kennicutt, R.C. Jr. 1983, ApJ, 272, 54

Kennicutt, R.C. Jr. 1984, ApJ, 287, 116

Kennicutt, R.C. Jr. 1998, ApJ, 498, 541

Kennicutt, R.C. Jr., \& Hodge, P.W. 1986, ApJ, 306, 130

Kennicutt, R.C. Jr., \& Skillman, E.D. 2001, AJ, 121, 1461

Kennicutt, R.C. Jr., Tamblyn, P., \& Congdon, C.W. 1994, ApJ, 435, 22

Klypin, A., Kravtsov, A. V., Valenzuela, O,, \& Prada, F. 1999, ApJ, 522, 82

Knezek, P. M., Sembach, K. R., \& Gallagher, J. S., III 1999, ApJ, 514, 119

Marlowe, A. T., Meurer, G. R., Heckman, T. M., \& Schommer, R. 1997, ApJS, 112, 285

Mateo, M. 1998, ARA\&A, 36, 435

Mayer, L., Governato, F., Colpi, M., Moore, B., Quinn, T., Wadsley, J., Stadel, J., \& Lake, G. 2001a, ApJ, 547, L123

Mayer, L., Governato, F., Colpi, M., Moore, B., Quinn, T., Wadsley, J., Stadel, J., \& Lake, G. 2001b, ApJ, 559, 754

Miller, B. W. 1994, Ph.D. Thesis, University of Washington

Miller, B. W. 1996, AJ, 112, 991

Miller, B. W., Dolphin, A. E., Lee, M. G., Kim, S. C., \& Hodge, P. 2001 ApJ, 562, 713

Miller, B.W., \& Hodge, P. 1994, ApJ, 427, 656

Moore, B., Ghigna, S., Governato, F., Lake, G., Quinn, T., Stadel, J., \& Tozzi, P. 1999, ApJ, 524, L19

Normandeau, M., Taylor, A.R., \& Dewdney, P.E. 1996, Nature, 380, 687

Oosterloo, T., Da Costa, G.S., \& Staveley-Smith, L. 1996, AJ, 112, 1969

Press, W. H., Teukolsky, S. A., Vetterling, W. T., \& Flannery, B. P. 1992, Numerical Recipes in Fortran, Cambridge University Press 
Puche, D., \& Carignan, C. 1988, AJ, 95, 1025

Quinn, T., Katz, N., \& Efstathiou, G. 1996, MNRAS, 278, L49

Richer, M. G. et al. 2001, A\&A, 370, 34

Roberts, M.S. 1963, ARA\&A, 1, 149

Sandage, A., \& Hoffman, G.L. 1991, ApJ, 379, 45

Scalo, J.M. 1986, Fund. Cos. Phys., 11, 1

Schlegel, D.J., Finkbeiner, D.P., \& Davis, M. 1998, ApJ, 500, 525

Skillman, E. D. 1996, ASP Conf. Ser. 106: The Minnesota Lectures on Extragalactic Neutral Hydrogen, 208

Skillman, E. D., Bomans, D. J., \& Kobulnicky, H. A. 1997, ApJ, 474, 205

St-Germain, J., Carignan, C., Côté, S., \& Oosterloo, T. 1999, AJ, 118, 1235

Tully, R.B., \& Fisher, J.R. 1987, Nearby Galaxies Atlas, Cambridge University Press

van den Bergh, S. 1994a, AJ, 107, 1328

van den Bergh, S. 1994b, ApJ, 428, 617

van den Bergh, S. 2000, PASP, 112, 529

van Zee, L. 2000, AJ, 119, 2757

van Zee, L. 2001, AJ, 121, 2003

van Zee, L., Haynes, M. P., \& Salzer, J. J. 1997a, AJ, 114, 2479

Whiting, A. B. 1999, AJ, 117, 202

Young, L.M., \& Lo, K.Y. 1997, ApJ, 490, 710

Youngblood, A.J., \& Hunter, D.A. 1999, ApJ, 519, 55 
Table 1. Sculptor Group Dwarf Irregular Galaxies with $\mathrm{H} \alpha$ Emission

\begin{tabular}{|c|c|c|c|c|c|c|}
\hline Galaxy Name & Alternate Name & R.A. (J2000) & dec. (J2000) & $v_{G S R}$ & $\mathrm{D}(\mathrm{Mpc})$ & $\mathrm{M}(\mathrm{B})$ \\
\hline $\mathrm{ESO} 347-\mathrm{G} 17^{\mathrm{a}}$ & & $23: 26: 57$ & $-37: 20: 47$ & 695 & 6.99 & -14.79 \\
\hline ESO $471-G 06^{a, b}$ & UGCA 442 & $23: 43: 47$ & $-31: 57: 09$ & 281 & 3.51 & -14.06 \\
\hline ESO 348-G09a & & $23: 49: 23$ & $-37: 46: 19$ & 640 & 6.52 & -13.75 \\
\hline SC 18 & & 00:00:59 & $-41: 09: 18$ & 119 & 2.14 & -9.20 \\
\hline NGC 59 & ESO 539-G04 & $00: 15: 25$ & $-21: 26: 36$ & 392 & 4.39 & -15.30 \\
\hline ESO $473-G 24^{a}$ & & $00: 31: 20$ & $-22: 46: 02$ & 571 & 5.94 & -12.68 \\
\hline $\mathrm{SC} 24$ & & $00: 36: 38$ & $-32: 34: 28$ & 61 & 1.66 & -8.39 \\
\hline DDO $226^{\mathrm{c}}$ & $\begin{array}{c}\text { UGCA } 9 \\
\text { IC } 1574\end{array}$ & 00:43:04 & $-22: 14: 49$ & 386 & 4.39 & -13.49 \\
\hline $\mathrm{DDO} 6^{\mathrm{c}}$ & $\begin{array}{l}\text { ESO } 474-G 18 \\
\text { UGCA } 15 \\
\text { ESO } 540-G 31\end{array}$ & $00: 49: 49$ & $-21: 00: 54$ & 318 & 3.82 & -12.24 \\
\hline AM 0106-382 & & 01:08:22 & $-38: 12: 33$ & 593 & 6.13 & -12.47 \\
\hline NGC $625^{\mathrm{a}}$ & ESO 297-G05 & $01: 35: 06$ & $-41: 26: 05$ & 332 & 3.93 & -16.31 \\
\hline ESO $245-G 05^{\mathrm{b}}$ & A 143 & 01:45:04 & $-43: 35: 53$ & 310 & 3.75 & -15.59 \\
\hline
\end{tabular}

${ }^{\mathrm{a}}$ observed spectroscopically in companion paper

${ }^{\mathrm{b}}$ not imaged in $\mathrm{H} \alpha$ here, observed by Miller (1996)

${ }^{\mathrm{c}}$ not imaged in $\mathrm{H} \alpha$ here, observed by van Zee (2000) 
Table 2. HII Regions Positions and $\mathrm{H} \alpha$ Fluxes

\begin{tabular}{|c|c|c|c|c|}
\hline Galaxy \& Number & \multicolumn{2}{|c|}{$(\mathrm{J} 2000)$} & $\begin{array}{c}\mathrm{H} \alpha \text { Flux } \\
10^{-15}{\mathrm{ergs} \mathrm{cm}^{-2} \mathrm{~s}^{-1}}^{-e^{2}}\end{array}$ & $\begin{array}{c}\log \mathrm{L}(\mathrm{H} \alpha) \\
\operatorname{ergs~s}^{-1}\end{array}$ \\
\hline ESO 347-G17 \#1 & $23: 26: 58.87$ & $-37: 20: 55.2$ & $19 \pm 4$ & 38.1 \\
\hline ESO $347-G 17 \# 2$ & $23: 26: 58.34$ & $-37: 20: 56.0$ & $2.1 \pm 0.4$ & 37.1 \\
\hline ESO 347-G17 \#3 & $23: 26: 58.00$ & $-37: 20: 54.3$ & $3.1 \pm 0.6$ & 37.3 \\
\hline ESO 347-G17 \#4 & $23: 26: 57.80$ & $-37: 20: 49.4$ & $1.6 \pm 0.3$ & 37.0 \\
\hline ESO 347-G17 \#5 & $23: 26: 57.13$ & $-37: 20: 49.4$ & $59 \pm 12$ & 38.6 \\
\hline ESO 347-G17 \#6 & $23: 26: 56.41$ & $-37: 20: 52.1$ & $2.3 \pm 0.5$ & 37.1 \\
\hline ESO 347-G17 \#7 & $23: 26: 56.44$ & $-37: 20: 39.3$ & $10.3 \pm 2.1$ & 37.8 \\
\hline ESO 347-G17 \#8 & $23: 26: 55.88$ & $-37: 20: 49.6$ & $4.9 \pm 1.0$ & 37.5 \\
\hline ESO 347-G17 \#9 & $23: 26: 55.61$ & $-37: 20: 50.6$ & $1.6 \pm 0.3$ & 37.0 \\
\hline ESO 347-G17 \#10 & $23: 26: 55.15$ & $-37: 20: 51.0$ & $25 \pm 5$ & 38.2 \\
\hline ESO 347-G17 \#11 & $23: 26: 53.36$ & $-37: 20: 51.9$ & $2.8 \pm 0.6$ & 37.2 \\
\hline ESO 348-G9 \#1 & $23: 49: 27.48$ & $-37: 46: 23.8$ & $1.0 \pm 0.2$ & 36.7 \\
\hline ESO $348-G 9 \# 2$ & $23: 49: 27.07$ & $-37: 46: 24.9$ & $0.4 \pm 0.1$ & 36.3 \\
\hline ESO 348-G9 \#3 & $23: 49: 26.52$ & $-37: 46: 16.9$ & $4.7 \pm 0.9$ & 37.4 \\
\hline ESO 348-G9 \#4 & $23: 49: 21.67$ & $-37: 46: 21.3$ & $0.7 \pm 0.1$ & 36.6 \\
\hline SC $18 \# 1$ & 00:00:58.02 & $-41: 09: 24.2$ & $5.3 \pm 0.5$ & 36.5 \\
\hline NGC $59 \# 1$ & $00: 15: 25.67$ & $-21: 26: 40.1$ & $26 \pm 5$ & 37.8 \\
\hline NGC $59 \# 2$ & $00: 15: 25.25$ & $-21: 26: 40.9$ & $130 \pm 26$ & 38.5 \\
\hline NGC $59 \# 3$ & $00: 15: 24.93$ & $-21: 26: 39.7$ & $4.9 \pm 1.0$ & 37.1 \\
\hline NGC $59 \# 4$ & $00: 15: 25.03$ & $-21: 26: 45.9$ & $1.9 \pm 0.4$ & 36.7 \\
\hline ESO 473-G24 \#1 & 00:31:23.12 & $-22: 46: 05.7$ & $9.4 \pm 1.9$ & 37.6 \\
\hline ESO 473-G24 \#2 & 00:31:22.16 & $-22: 46: 14.6$ & $12.3 \pm 2.5$ & 37.7 \\
\hline ESO 473-G24 \#3 & 00:31:22.02 & $-22: 46: 18.2$ & $8.9 \pm 1.8$ & 37.6 \\
\hline ESO 473-G24 \#4 & 00:31:21.28 & $-22: 46: 14.1$ & $7.0 \pm 1.4$ & 37.5 \\
\hline $\mathrm{SC} 24 \# 1$ & 00:36:38.35 & $-32: 34: 45.9$ & $0.8 \pm 0.1$ & 35.4 \\
\hline AM 0106-382 \#1 & 01:08:21.87 & $-38: 12: 33.7$ & $\cdots$ & $\cdots$ \\
\hline
\end{tabular}


Table 2-Continued

\begin{tabular}{|c|c|c|c|c|}
\hline Galaxy \& Number & \multicolumn{2}{|c|}{$(\mathrm{J} 2000)$} & $\begin{array}{c}\mathrm{H} \alpha \text { Flux } \\
10^{-15} \mathrm{ergs}_{\mathrm{cm}^{-2} \mathrm{~s}^{-1}}\end{array}$ & $\begin{array}{c}\log \mathrm{L}(\mathrm{H} \alpha) \\
\operatorname{ergs~s}^{-1}\end{array}$ \\
\hline AM 0106-382 \#2 & 01:08:22.57 & $-38: 12: 35.6$ & $\ldots$ & $\ldots$ \\
\hline AM 0106-382 \#3 & 01:08:22.33 & $-38: 12: 42.5$ & $\ldots$ & $\ldots$ \\
\hline AM 0106-382 \#4 & 01:08:21.23 & $-38: 12: 42.6$ & $\cdots$ & $\cdots$ \\
\hline AM 0106-382 \#5 & 01:08:21.13 & $-38: 12: 45.9$ & $\cdots$ & $\cdots$ \\
\hline NGC $625 \# 1$ & 01:35:07.36 & $-41: 26: 11.2$ & $30 \pm 3$ & 37.8 \\
\hline NGC $625 \# 2$ & 01:35:07.23 & $-41: 25: 52.5$ & $1.4 \pm 0.1$ & 36.4 \\
\hline NGC $625 \# 3$ & 01:35:06.78 & $-41: 26: 00.1$ & $15.3 \pm 1.5$ & 37.5 \\
\hline NGC $625 \# 4$ & 01:35:06.86 & $-41: 26: 05.9$ & $193 \pm 19$ & 38.6 \\
\hline NGC $625 \# 5$ & 01:35:06.68 & $-41: 26: 13.0$ & $2100 \pm 210$ & 39.6 \\
\hline NGC $625 \# 6$ & 01:35:06.53 & $-41: 26: 19.8$ & $51 \pm 5$ & 38.0 \\
\hline NGC $625 \# 7$ & 01:35:06.34 & $-41: 25: 52.8$ & $14.1 \pm 1.4$ & 37.4 \\
\hline NGC $625 \# 8$ & 01:35:06.11 & $-41: 26: 04.2$ & $23.0 \pm 2.3$ & 37.6 \\
\hline NGC $625 \# 9$ & 01:35:05.85 & $-41: 26: 11.2$ & $470 \pm 47$ & 39.0 \\
\hline NGC $625 \# 10$ & 01:35:05.86 & $-41: 25: 59.6$ & $22.4 \pm 2.2$ & 37.6 \\
\hline NGC $625 \# 11$ & 01:35:05.73 & $-41: 25: 44.3$ & $12.0 \pm 1.2$ & 37.4 \\
\hline NGC $625 \# 12$ & 01:35:05.20 & $-41: 25: 49.4$ & $7.5 \pm 0.8$ & 37.2 \\
\hline NGC $625 \# 13$ & 01:35:05.36 & $-41: 26: 07.5$ & $19.3 \pm 1.9$ & 37.6 \\
\hline NGC $625 \# 14$ & 01:35:05.54 & $-41: 26: 15.5$ & $32 \pm 3$ & 37.8 \\
\hline NGC $625 \# 15$ & 01:35:05.26 & $-41: 26: 12.4$ & $32 \pm 3$ & 37.8 \\
\hline NGC $625 \# 16$ & 01:35:05.16 & $-41: 26: 15.1$ & $14.1 \pm 1.4$ & 37.4 \\
\hline NGC $625 \# 17$ & 01:35:04.59 & $-41: 26: 18.1$ & $21.1 \pm 2.1$ & 37.6 \\
\hline NGC $625 \# 18$ & 01:35:04.80 & $-41: 26: 09.3$ & $161 \pm 16$ & 38.5 \\
\hline NGC $625 \# 19$ & 01:35:04.64 & $-41: 26: 01.4$ & $4.7 \pm 0.5$ & 37.0 \\
\hline NGC $625 \# 20$ & 01:35:04.42 & $-41: 26: 00.9$ & $2.6 \pm 0.3$ & 36.7 \\
\hline NGC $625 \# 21$ & 01:35:03.99 & $-41: 26: 07.3$ & $7.6 \pm 0.8$ & 37.2 \\
\hline NGC $625 \# 22$ & 01:35:01.96 & $-41: 26: 25.8$ & $23.0 \pm 2.3$ & 37.6 \\
\hline NGC $625 \# 23$ & 01:35:01.47 & $-41: 26: 25.9$ & $19.3 \pm 1.9$ & 37.6 \\
\hline
\end{tabular}


Table 3. Star Formation Properties of Sculptor Group dI Galaxies

\begin{tabular}{lcccccc}
\hline \hline \multicolumn{1}{c}{ Galaxy } & $\begin{array}{c}\text { SFR } \\
\mathrm{M}_{\odot} \mathrm{yr}^{-1}\end{array}$ & $\begin{array}{c}\mathrm{SFR} / \mathrm{L}(\mathrm{B}) \\
\mathrm{M}_{\odot} \mathrm{yr}^{-1} \mathrm{~L}_{\odot}^{-1}\end{array}$ & $\begin{array}{c}\tau_{\text {form }} \\
\mathrm{Gyr}\end{array}$ & $\begin{array}{c}\mathrm{M}(\mathrm{HI})^{\mathrm{a}} \\
10^{6} \mathrm{M}_{\odot}\end{array}$ & $\begin{array}{c}\mathrm{M}(\mathrm{HI}) / \mathrm{L}(\mathrm{B}) \\
\mathrm{M}_{\odot} / \mathrm{L}_{\odot}\end{array}$ & $\begin{array}{c}\tau_{\text {gas }}{ }^{\mathrm{b}} \\
\mathrm{Gyr}\end{array}$ \\
\hline ESO 347-G17 & $6.3 \times 10^{-3}$ & $5.3 \times 10^{-11}$ & 19 & 120 & 1.00 & 25 \\
ESO 471-G06 & $2.0 \times 10^{-3}$ & $3.3 \times 10^{-11}$ & 30 & 163 & 2.66 & 110 \\
ESO 348-G09 & $2.8 \times 10^{-4}$ & $0.6 \times 10^{-11}$ & 167 & 84.3 & 1.83 & 400 \\
SC 18 & $2.4 \times 10^{-5}$ & $3.4 \times 10^{-11}$ & 29 & 5.0 & 7.13 & 270 \\
NGC 59 & $3.1 \times 10^{-3}$ & $1.6 \times 10^{-11}$ & 63 & 16.7 & 0.87 & 7.1 \\
ESO 473-G24 & $1.3 \times 10^{-3}$ & $7.6 \times 10^{-11}$ & 13 & 63.8 & 3.71 & 65 \\
SC 24 & $2.1 \times 10^{-6}$ & $0.6 \times 10^{-11}$ & 167 & 2.1 & 6.29 & 1300 \\
DDO 226 & $1.3 \times 10^{-3}$ & $3.6 \times 10^{-11}$ & 28 & 33.9 & 0.93 & 36 \\
DDO 6 & $2.9 \times 10^{-4}$ & $2.5 \times 10^{-11}$ & 40 & 15.4 & 1.34 & 70 \\
NGC 625 & $5.0 \times 10^{-2}$ & $10.2 \times 10^{-11}$ & 9.8 & 118 & 0.24 & 3.1 \\
ESO 245-G05 & $1.0 \times 10^{-2}$ & $3.9 \times 10^{-11}$ & 26 & 289 & 1.15 & 38 \\
\hline
\end{tabular}

aTotal galaxy HI mass from Côté (1995), adjusted to the distance in Table 1

${ }^{\mathrm{b}} \tau_{\text {gas }}$ is the gas depletion time scale $=($ Total Gas Mass $) /(\mathrm{SFR})$, where the total gas mass is $1.32 \times \mathrm{M}(\mathrm{HI})$ to account for $\mathrm{He}$ 
A complete gzipped .ps file containing Figure 1 can be obtained via anonymous ftp from ftp.astro.umn.edu /pub/users/skillman/sculptor in paper1.ps.gz

Fig. 1.- Images of 8 Sculptor Group dwarf irregular galaxies. The original $\mathrm{H} \alpha$ images of the galaxies are shown in the left panels and the continuum ( $i$-band) subtracted H $\alpha$ images are shown in the right panels. The field of view for all of the images is identical at $301 \times 301$ pixels or $1.96^{\prime}$ $\times 1.96^{\prime}$. The HII regions are labeled. Astrometric positions and $\mathrm{H} \alpha$ fluxes are listed in Table 2 . 


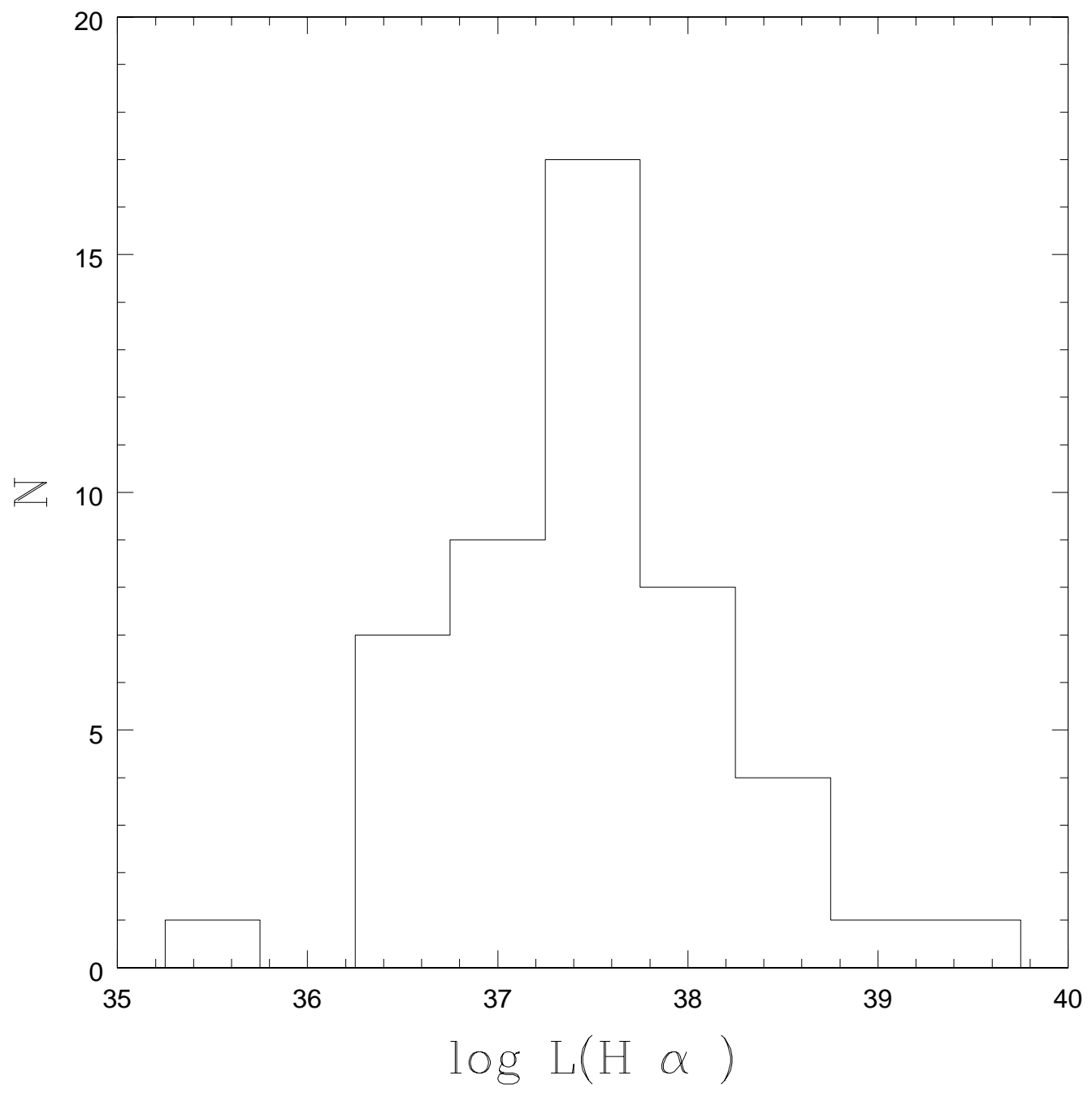

Fig. 2.- A histogram of the HII region luminosities for the 8 Sculptor Group dIs observed here in $\mathrm{H} \alpha$. 


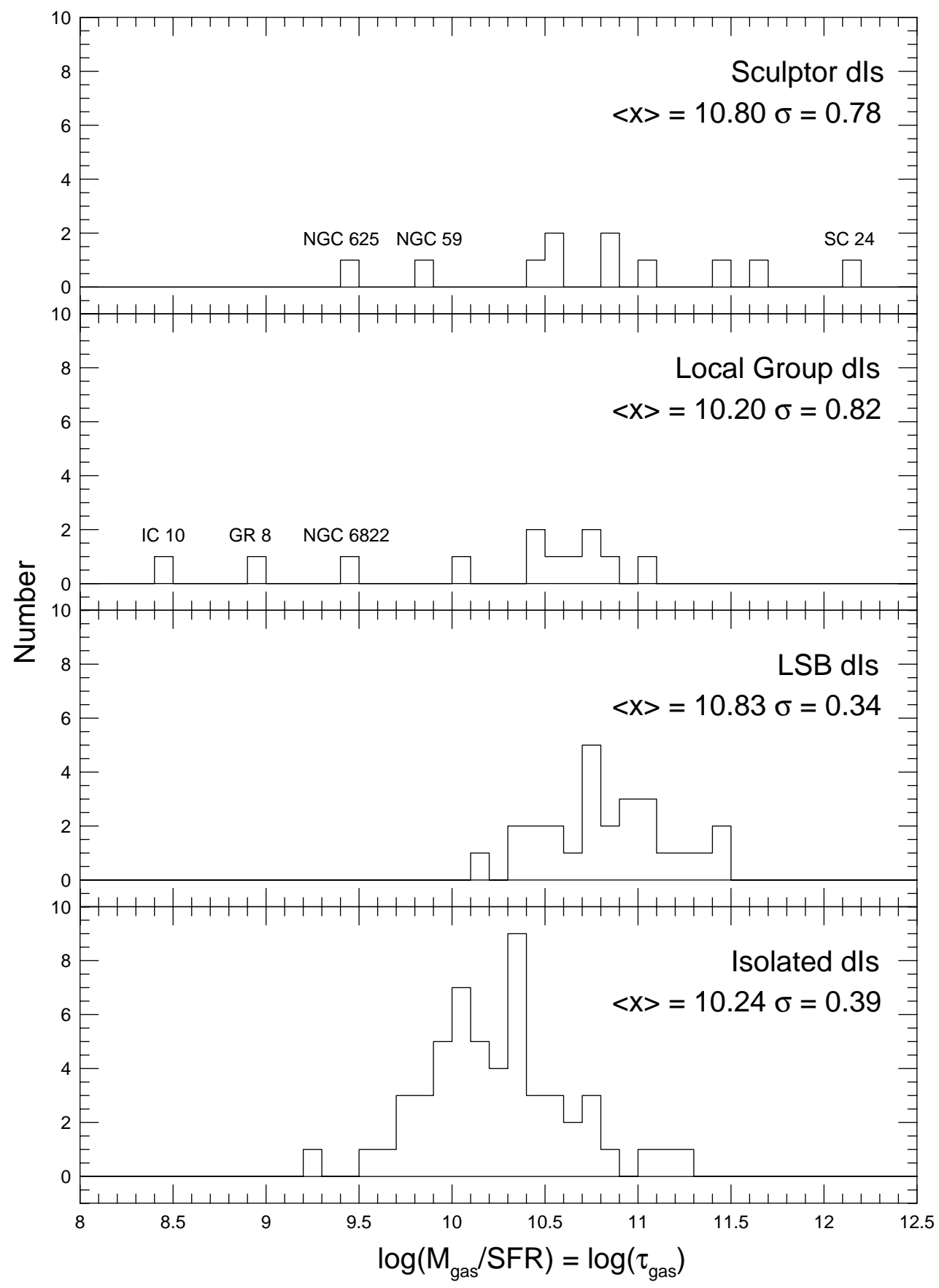

Fig. 3. - A comparison of the ratio of the gas mass to the current star formation rate $\left(=\tau_{\text {gas }}\right)$ for the Sculptor Group dIs and three comparison groups: the Local Group dIs (from Mateo 1998), the gas-rich LSB galaxies studied by van Zee et al. (1997), and the isolated dIs of van Zee (2000, 2001). For each sample, the mean value and the standard deviation in the sample is given. 


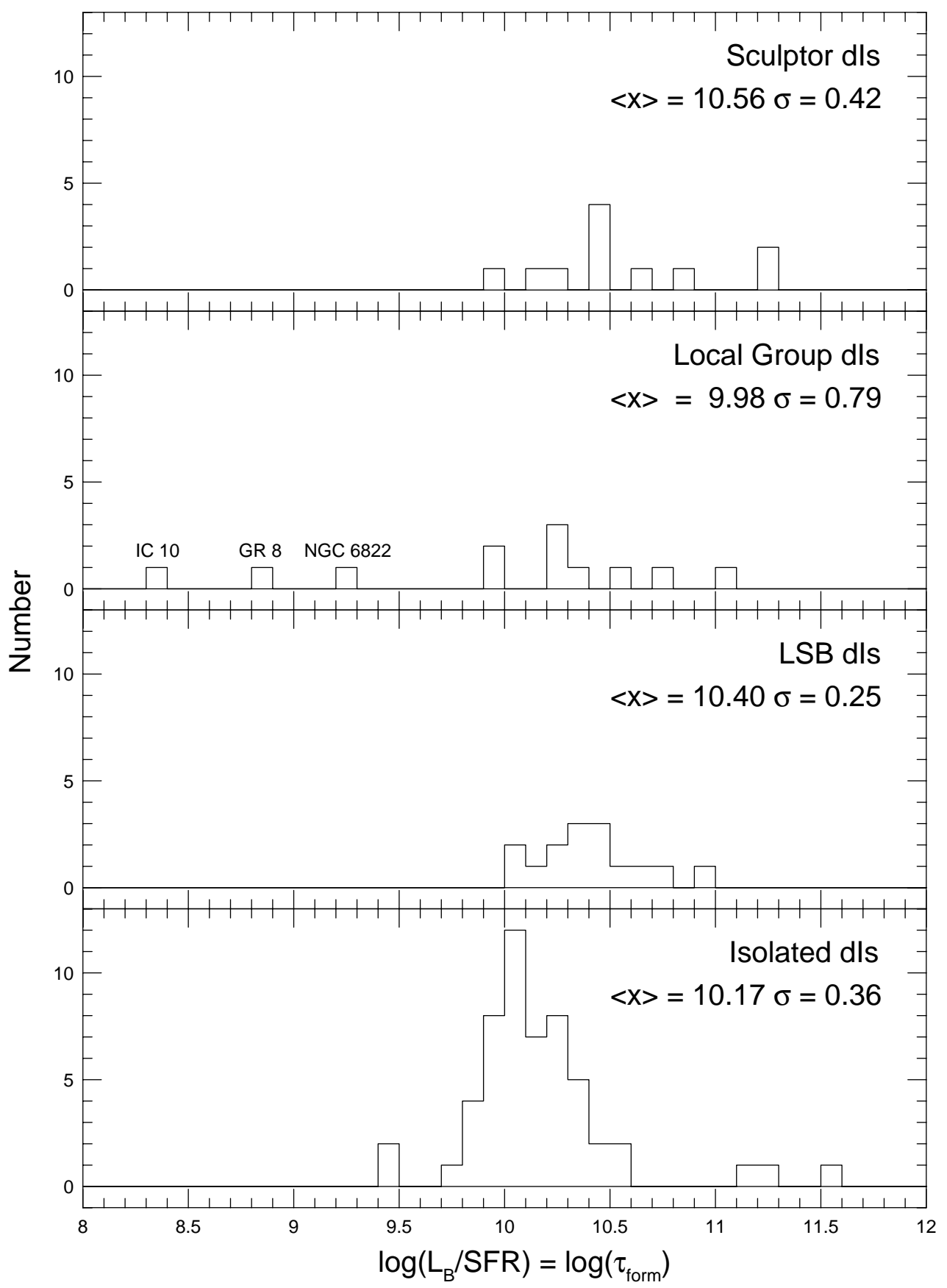

Fig. 4. - A comparison of the ratio of the luminosity to the current star formation rate $\left(=\tau_{\text {form }}\right)$ for the Sculptor Group dIs and three comparison groups: the Local Group dIs (from Mateo 1998), the gas-rich LSB galaxies studied by van Zee et al. (1997), and the isolated dIs of van Zee (2000, 2001). For each sample, the mean value and the standard deviation in the sample is given. 


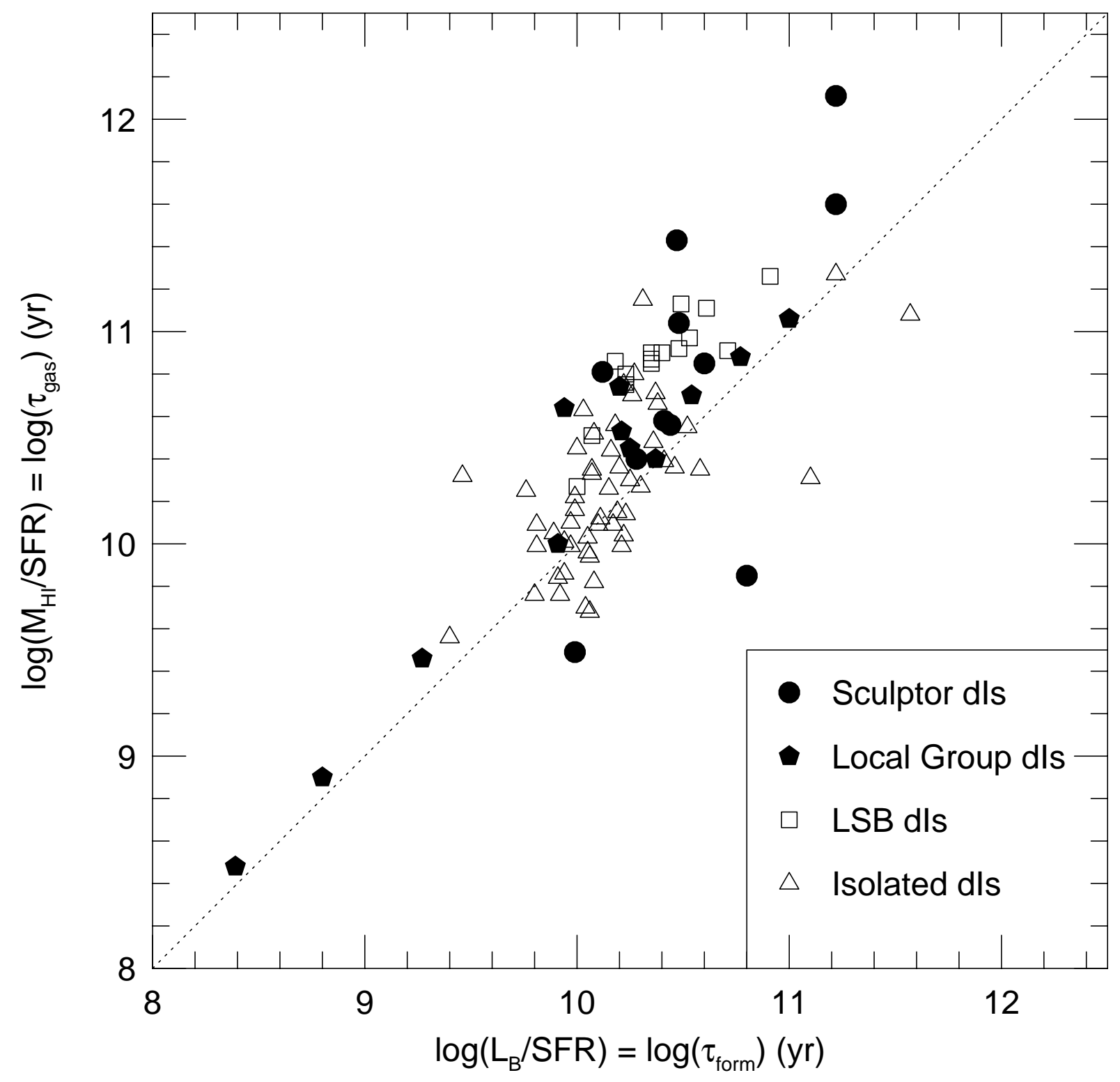

Fig. 5. - A comparison of the ratio of the gas mass to the current star formation rate $\left(=\tau_{\text {gas }}\right)$ to the ratio of the luminosity to the current star formation rate $\left(=\tau_{\text {form }}\right)$ for the Sculptor Group dIs and three comparison groups: the Local Group dIs (from Mateo 1998), the gas-rich LSB galaxies studied by van Zee et al. (1997), and the isolated dIs of van Zee (2000, 2001). The dotted line represents an equality between $\tau_{\text {gas }}$ and $\tau_{\text {form }}$, or, equivalently, $\mathrm{M}(\mathrm{HI}) / \mathrm{L}(\mathrm{B})=1.0$. 


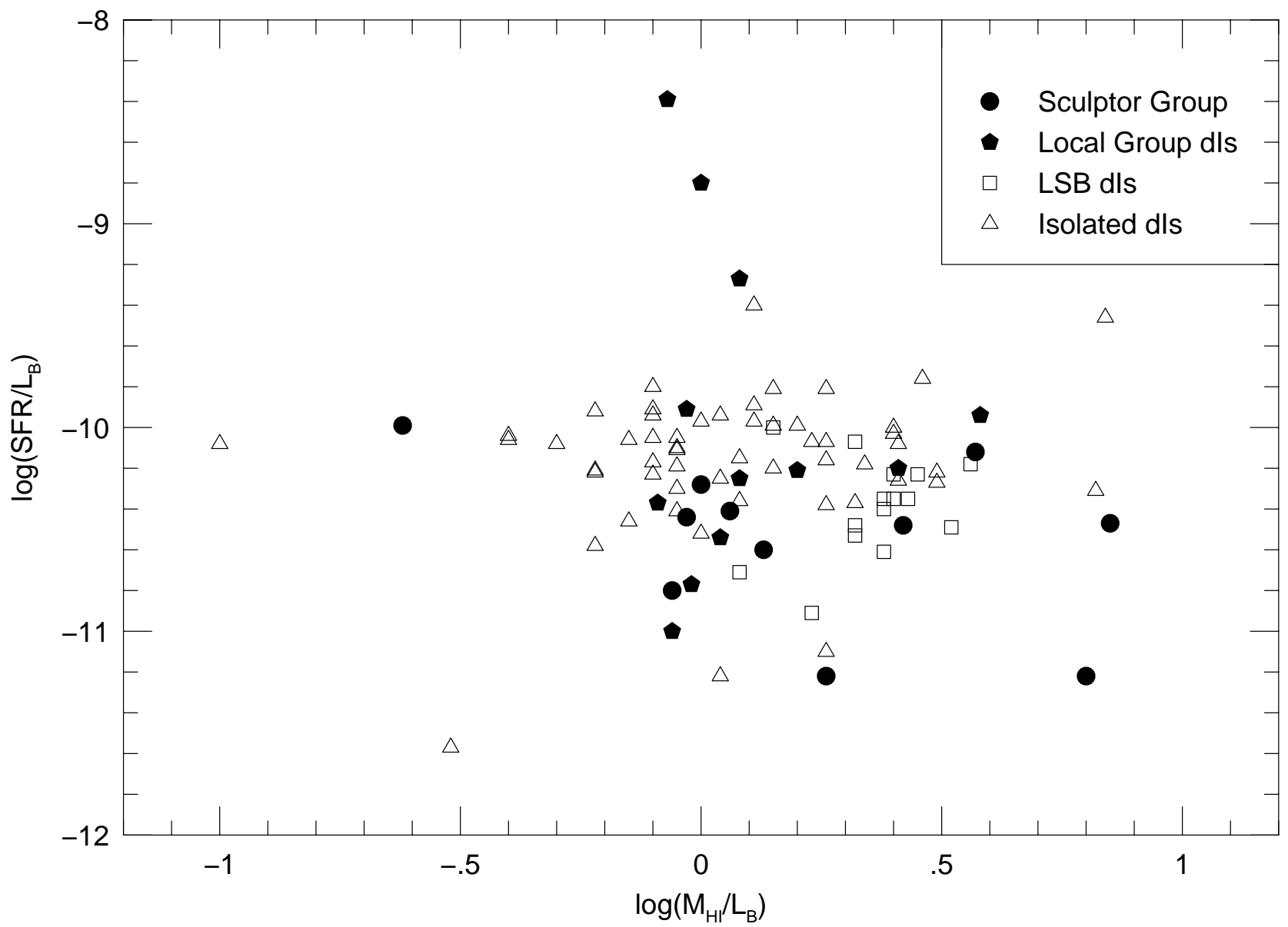

Fig. 6.- A comparison of SFR and gas mass normalized to the galaxy luminosity the Sculptor Group dIs and three comparison groups: the Local Group dIs (from Mateo 1998), the gas-rich LSB galaxies studied by van Zee et al. (1997), and the isolated dIs of van Zee (2000, 2001). 

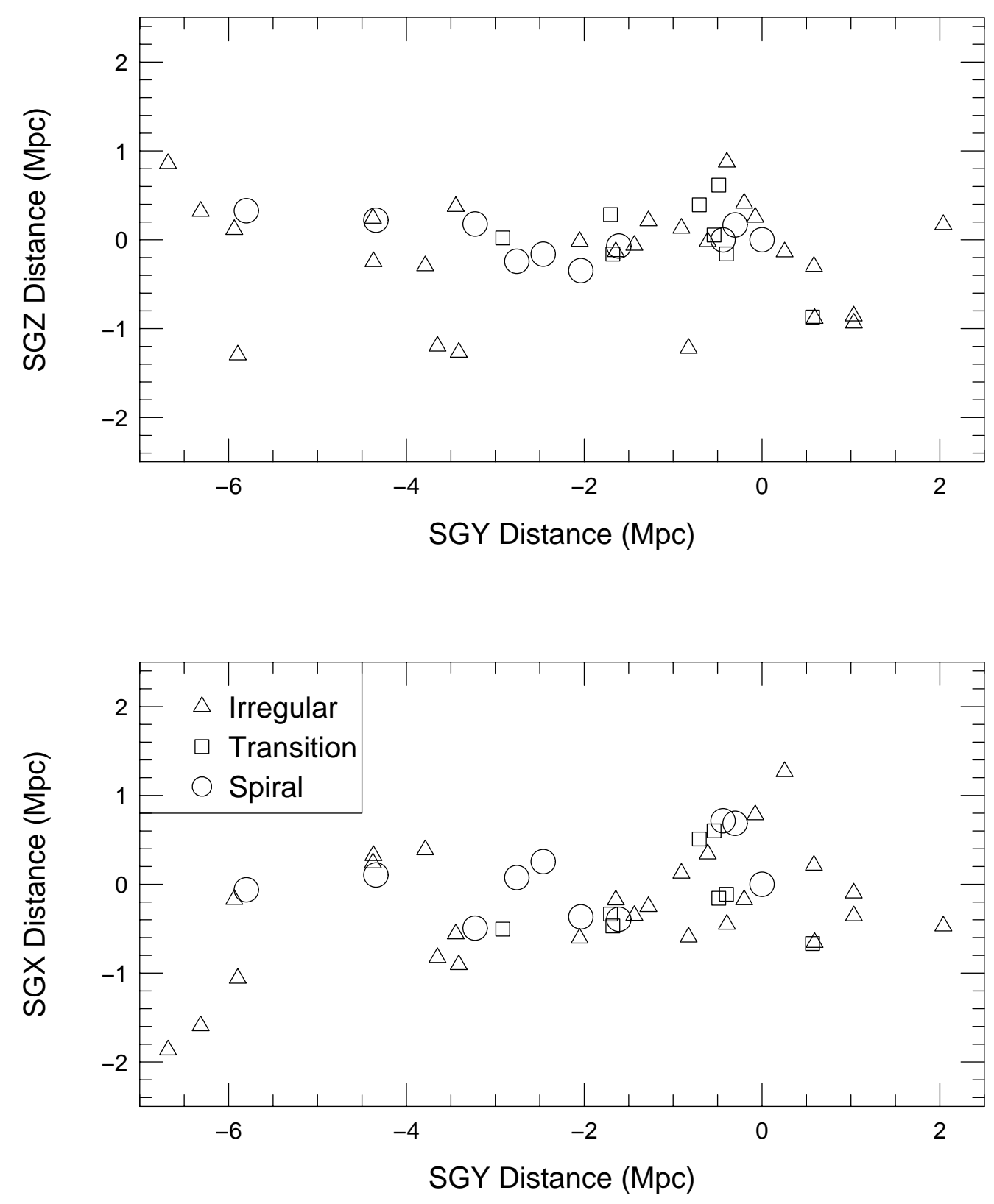

Fig. 7.- A comparison of the positions of the spirals (circles), dIs (triangles), and transition type galaxies (squares) in the Local Group and Sculptor Group plotted in super-galactic coordinates Z versus $\mathrm{Y}$ and $\mathrm{X}$ versus Y. The Local Group and the Sculptor Group appear to join smoothly when portrayed in this manner (see also Tully \& Fisher 1987 and Figure 9 of Jerjen et al. 1998). 


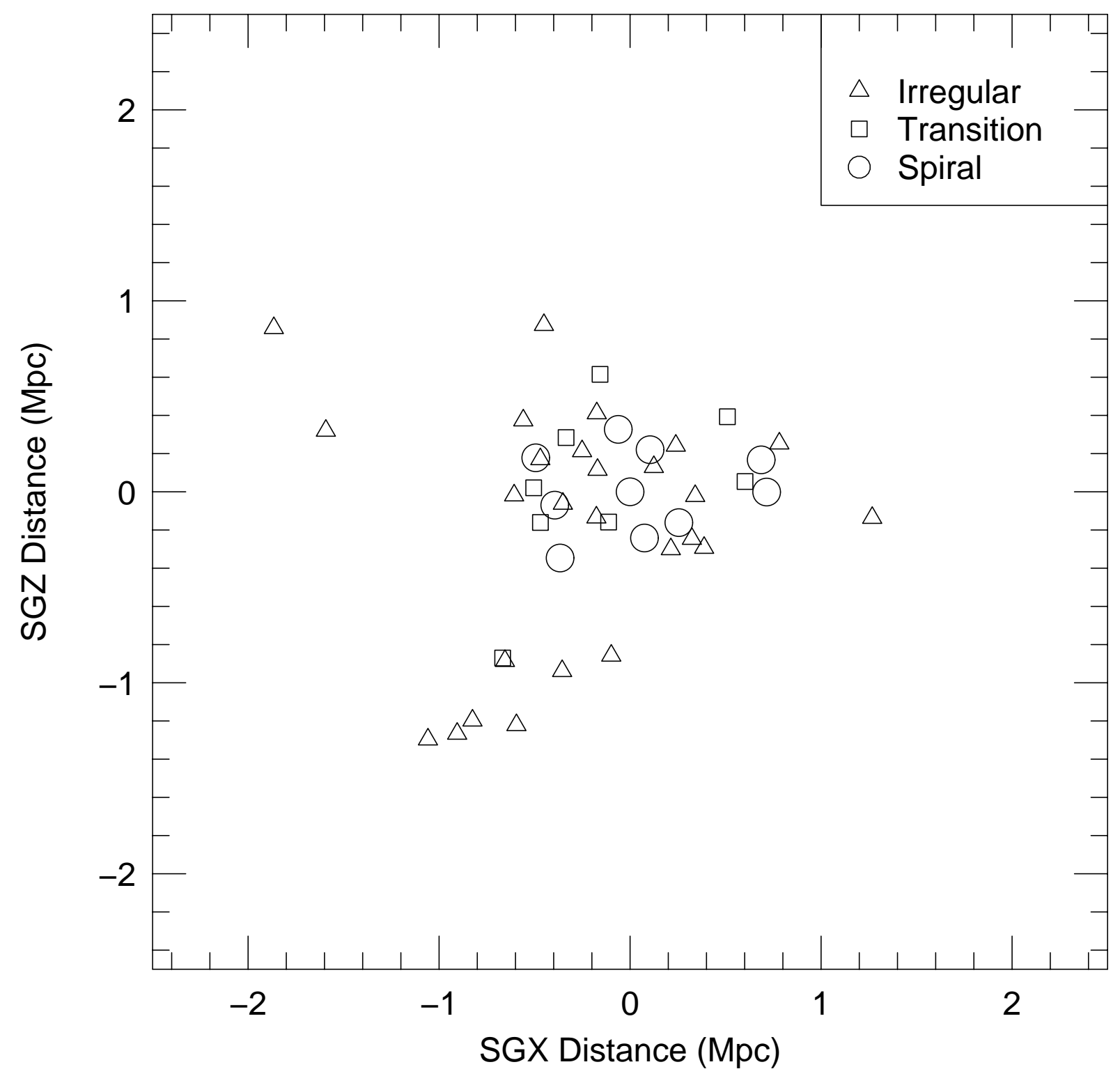

Fig. 8.- A comparison of the positions of the spirals (circles), dIs (triangles), and transition type galaxies (squares) in the Local Group and Sculptor Group plotted in super-galactic X and Z coordinates. The dIs lie at preferentially larger radii in this projection. 


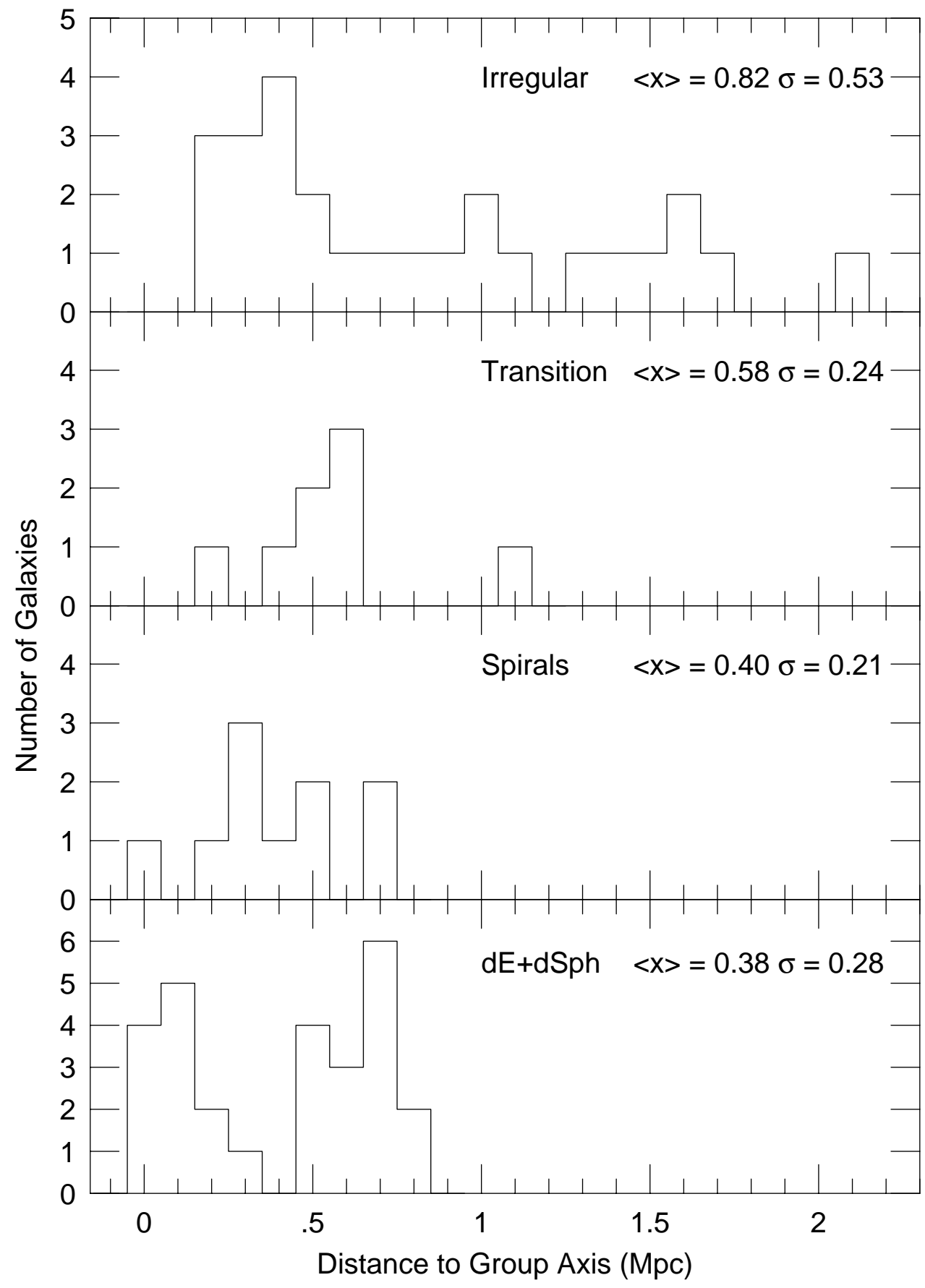

Fig. 9.- A histogram comparison of the radial distances of the dIs, transition, spiral, and dE type galaxies in the Local Group and Sculptor Group concentration (as described in the text and shown in Figure 8). For each sample, the mean value and the standard deviation in the sample is given. On average, the dIs lie at preferentially larger radii and the transition galaxy distances are comparable to those of the spiral and $\mathrm{dE}$ galaxies. 


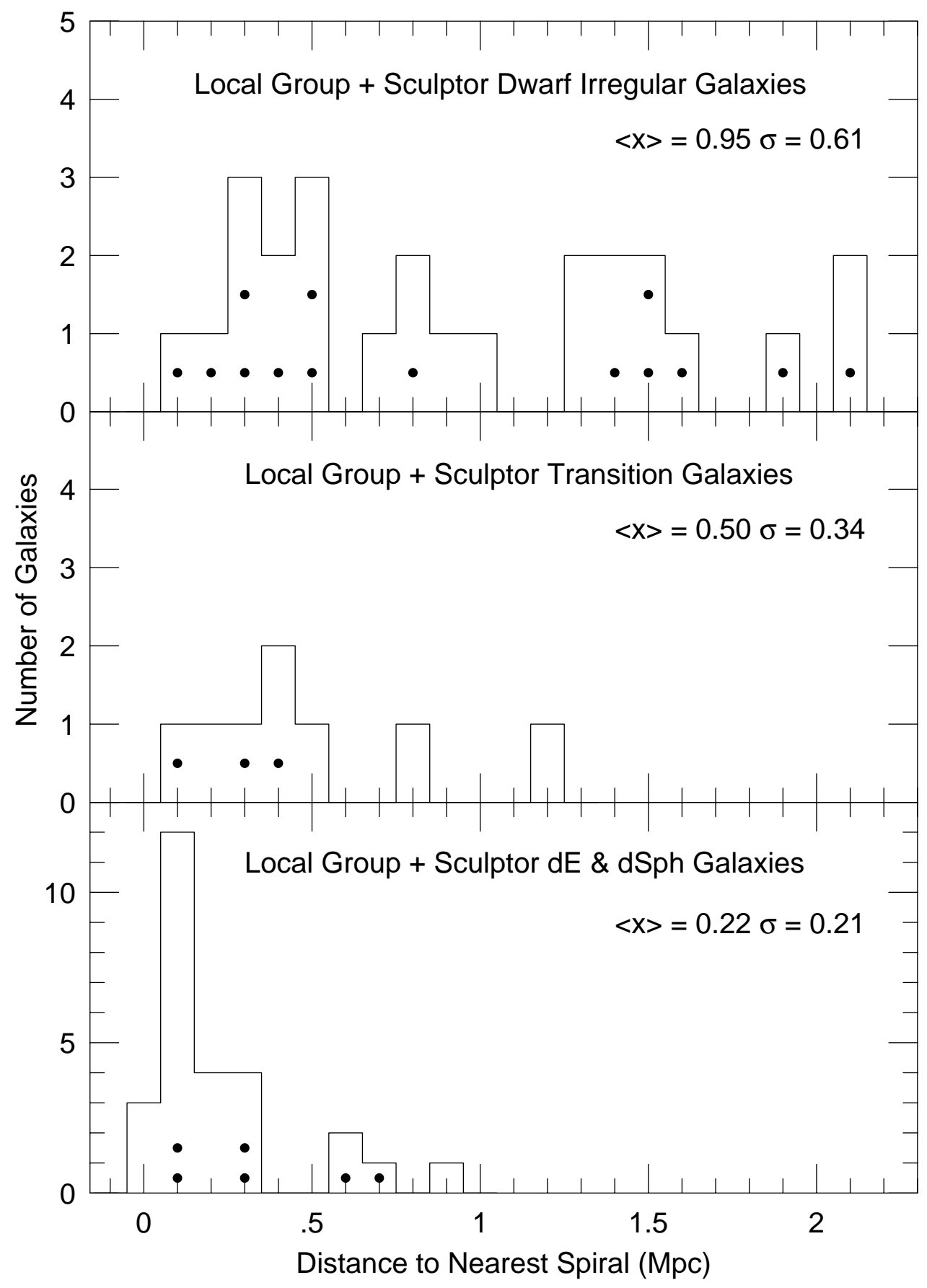

Fig. 10.- A histogram comparison of the distances to the nearest spiral galaxy for the dI, transition type, and dE galaxies in the Local Group and Sculptor Group. For each sample, the mean value and the standard deviation in the sample is given. On average, the dIs lie at preferentially larger distances from the spiral galaxies than the transition galaxies, and the transition galaxies are at larger distances than the dEs. The dots indicate the Sculptor Group members (see text). 
This figure "Skillman.fig1a.jpg" is available in "jpg" format from: http://arxiv.org/ps/astro-ph/0211447v1 
This figure "Skillman.fig1b.jpg" is available in "jpg" format from: http://arxiv.org/ps/astro-ph/0211447v1 
This figure "Skillman.fig1c.jpg" is available in "jpg" format from: http://arxiv.org/ps/astro-ph/0211447v1 
This figure "Skillman.fig1d.jpg" is available in "jpg" format from: http://arxiv.org/ps/astro-ph/0211447v1 
This figure "Skillman.fig1e.jpg" is available in "jpg" format from: http://arxiv.org/ps/astro-ph/0211447v1 
This figure "Skillman.fig1f.jpg" is available in "jpg" format from: http://arxiv.org/ps/astro-ph/0211447v1 
This figure "Skillman.fig1g.jpg" is available in "jpg" format from: http://arxiv.org/ps/astro-ph/0211447v1 
This figure "Skillman.fig1h.jpg" is available in "jpg" format from: http://arxiv.org/ps/astro-ph/0211447v1 
This figure "Skillman.fig1i.jpg" is available in "jpg" format from: http://arxiv.org/ps/astro-ph/0211447v1 
This figure "Skillman.fig1j.jpg" is available in "jpg" format from: http://arxiv.org/ps/astro-ph/0211447v1 
This figure "Skillman.fig1k.jpg" is available in "jpg" format from: http://arxiv.org/ps/astro-ph/0211447v1 
This figure "Skillman.fig11.jpg" is available in "jpg" format from: http://arxiv.org/ps/astro-ph/0211447v1 
This figure "Skillman.fig1o.jpg" is available in "jpg" format from: http://arxiv.org/ps/astro-ph/0211447v1 
This figure "Skillman.fig1p.jpg" is available in "jpg" format from: http://arxiv.org/ps/astro-ph/0211447v1 